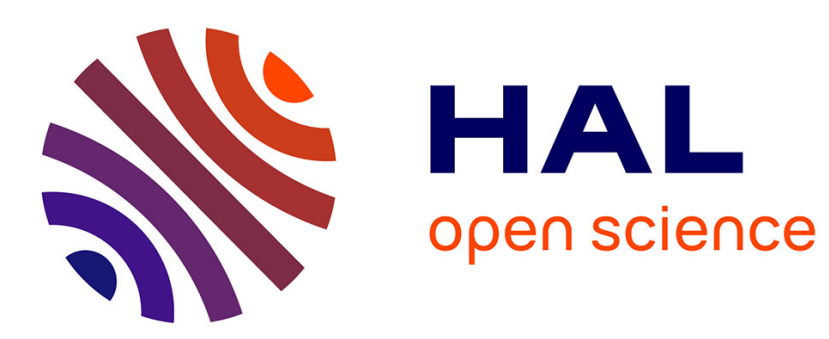

\title{
Clocks in Space for Tests of Fundamental Physics
}

Pacôme Delva, A. Hees, Peter Wolf

\section{To cite this version:}

Pacôme Delva, A. Hees, Peter Wolf. Clocks in Space for Tests of Fundamental Physics. Space Science Reviews, 2017, pp.1-37. 10.1007/s11214-017-0361-9 . hal-01590802

\section{HAL Id: hal-01590802 https://hal.sorbonne-universite.fr/hal-01590802}

Submitted on 20 Sep 2017

HAL is a multi-disciplinary open access archive for the deposit and dissemination of scientific research documents, whether they are published or not. The documents may come from teaching and research institutions in France or abroad, or from public or private research centers.
L'archive ouverte pluridisciplinaire HAL, est destinée au dépôt et à la diffusion de documents scientifiques de niveau recherche, publiés ou non, émanant des établissements d'enseignement et de recherche français ou étrangers, des laboratoires publics ou privés. 


\title{
Clocks in Space for Tests of Fundamental Physics
}

\author{
Pacôme Delva ${ }^{1}$, Aurélien Hees ${ }^{2,3}$, Peter Wolf ${ }^{1}$ \\ ${ }^{1}$ SYRTE, Observatoire de Paris, PSL Research University, CNRS, Sorbonne Universités, UPMC \\ Univ. Paris 06, LNE, 75014 Paris, France \\ ${ }^{2}$ Department of Mathematics, Rhodes University, 6140 Grahamstown, South Africa \\ ${ }^{3}$ Department of Physics and Astronomy, University of California, Los Angeles, CA 90095
}

\section{Introduction}

In our present understanding, and at its most fundamental level, physics is based on two theories: the Standard Model of particle physics (SM) that describes electromagnetism and the (strong and weak) nuclear interactions, and General Relativity (GR) that accounts for all gravitational phenomena. In spite of the overwhelming success of these two theories in describing much of the observed universe, a number of open issues, both theoretical and experimental remain. The assumed validity of GR at cosmological scales, together with a hypothesis of homogeneity and isotropy, have led to the "concordance model" of cosmology, referred to as the $\Lambda$ CDM model, which is in agreement with all present-day observations at large scales, notably the most recent observations of the anisotropies of the cosmic microwave background by the Planck satellite. However, important difficulties remain, in particular the necessary introduction of dark energy, described by a cosmological constant $\Lambda$, and of cold dark matter, made of some unknown, stable particle, which is not accounted for in the SM.

On the theoretical side, the SM is a quantum field theory, whilst GR, as well as many other alternative theories of gravitation, are classical. As such, they are fundamentally incomplete, because they do not include quantum effects. Most physicists agree that GR and SM are only low-energy approximations of a more fundamental theory that remains to be discovered, and that would provide a unified description of all interactions. Most attempts at such a unified theory lead to tiny violations of the basic principles of $G R$ and/or the $S M$, in particular the Einstein Equivalence Principle (EEP), at a, in general unknown, level of accuracy. It is the aim of highaccuracy fundamental physics experiments, like the ones described here, to search for first experimental hints of such modifications by making use of the outstanding performance provided by modern time/frequency metrology.

Indeed, of all quantities in the SI system of units, time is the one that can be measured with by far the smallest uncertainty in relative value. Best present day clocks reach relative uncertainties in the low $10^{-18}$ range and progress is rapid with no obvious hard limit in sight. One of the first and most challenging applications of such clocks are the experimental study of fundamental physics, and in particular the two fundamental theories mentioned above (GR and SM). As in GR space-time and gravitation are intimately related, it is no surprise that such high performance 
clocks, when compared over large distances and in varying gravitational fields, are one of the prime tools in the study of gravitation, GR and fundamental physics in general. However, large distances and large variations of gravitational fields are the realm of space science, and therefore putting high performance clocks into space, whilst still a technological challenge, certainly promises highly rewarding results in terms of fundamental physics.

In this chapter we describe some of the past, present and near future experiments that take advantage of clocks in space. It is impossible to describe all missions and projects in the field, and we will only mention some of the most important ones. Nonetheless, we hope that the reader will learn from the past, appreciate the present and look forward to the future of space clocks in fundamental physics.

\section{Theoretical background}

The classical theory of General Relativity provides a geometrical description of the gravitational interaction. It is based on two fundamental principles: (i) the Einstein Equivalence Principle (EEP) and (ii) the Einstein field equations that can be derived from the Einstein-Hilbert action. All GR extensions or alternative theories of gravitation will break at least one of these principles.

\subsection{The Einstein Equivalence Principle}

The first part of GR, the EEP, published in 1911 by A. Einstein (Einstein, 1911), gives gravitation a geometric nature. This principle implies that gravity can be identified to space-time geometry which is described mathematically by a symmetric order 2 tensor, the space-time metric $g_{\mu \nu}$. More precisely, the EEP implies that there exists only one space-time metric to which all matter minimally couples to (see Thorne et al, 1971). In practice, this means that the equations of motion for matter can be derived from the action of the Standard Model of particles in which the Minkowski metric $\eta_{\mu \nu}$ is replaced by the space-time metric $g_{\mu \nu}$ :

$$
S_{m a t}=\int d^{4} x \sqrt{-g} L_{m a t}\left(g_{\mu \nu}, \psi\right)
$$

where $g$ is the determinant of the space-time metric and $L_{m a t}\left(g_{\mu \nu}, \psi\right)$ is the standard Lagrangian for matter depending on the matter fields $\psi$.

From a theoretical point of view, this part of Einstein's theory allows one to derive the effects of gravitation from the space-time curvature. In particular, this implies that test bodies follow geodesics of this space-time. Furthermore, ideal clocks will measure the quadratic invariant of the space-time metric $d \tau^{2}=-g_{\mu \nu} d x^{\mu} d x^{\nu}$. Similarly, the propagation of electromagnetic waves is governed by Maxwell equations in which standard derivatives are replaced by covariant derivatives. At the geometric optics approximation, this implies that light rays are described by null geodesics. 
From a phenomenological point of view, three aspects of the EEP can be tested (see Will, 1993 and Will 2014): (i) the Universality of Free Fall (UFF), (ii) the Local Lorentz Invariance (LLI) and (iii) the Local Position Invariance (LPI). Furthermore, the Schiff conjecture stipulates that any complete, self-consistent theory of gravity that embodies the UFF necessarily embodies the two other parts of the EEP: the LPI and the LLI (see e.g. Will, 2014). However, a precise and general quantitative relationship between the sub-principles is unknown. It depends on the underlying model and varies between different candidate theories. Furthermore, no complete proof of this conjecture has been proposed and some counter-examples are known (see the discussion in Section 2.1.1 of Will, 2014).

\section{a) The Universality of Free Fall}

The UFF states that the motion of a test body is independent of its composition. It has been constrained with various experiments. Precise tests of the UFF compare the free fall accelerations, $a_{1}$ and $a_{2}$, of two different test bodies 1 and 2 falling in the gravitational field sourced by a body S. A succinct expression for the test of the UFF takes the form (see Will, 1993 and Will, 2014)

$$
\left(\frac{\Delta a}{a}\right)_{S ; 1-2}=2 \frac{a_{1}-a_{2}}{a_{1}+a_{2}} \approx\left(\frac{m_{P}}{m_{A}}\right)_{1}-\left(\frac{m_{P}}{m_{A}}\right)_{2}
$$

where $m_{P}$ and $m_{A}$ are the passive and active masses of each body. The UFF has been tested in various cases. First, it has been tested by comparing the motion of two different macroscopic bodies of standard matter. The best current constraints on the relative differential acceleration of two bodies are at the level of $10^{-13}$ and have been obtained by using Lunar Laser Ranging observations (see Williams et al, 2009 and 2012) and using torsion balances (see Schlamminger et al, 2008 and Adelberger et al, 2009). More recently, the UFF has been tested by comparing the acceleration measured by a macroscopic mass with the acceleration measured by a microscopic, quantum system and also by comparing the accelerations measured by two different microscopic quantum systems. This has been achieved using atom interferometry. The current constraints on the UFF between macroscopic and microscopic systems are at the level of $10^{-9}$ (see Peters, 1999). Similar constraints using two different types of atom interferometers have provided constraints at the level of $10^{-9}$ as well (see Peters et al, 2001, Merlet et al, 2010 and Zhou et al, 2015). Furthermore, there is a priori no reason that bodies with different spins react to gravity in a similar way. Therefore, the UFF has also been tested by considering atoms with different spins and the related constraints are at the level of $10^{-7}$ (see Tarallo et al, 2014). One can wonder if anti-matter falls any differently than standard matter. Tests of the UFF using anti-hydrogen atoms are currently on-going at CERN with the AEgIS (see Aghion et al, 2013) and GBAR (see Perez et al, 2012) experiments.

Even though the existing phenomenological tests of the UFF are quite extensive for standard matter, the question of the validity of the EEP in the dark sector remains open. Since dark matter has not been directly detected so far, tests of the EEP in the dark sector are model dependent. A lot of models introduced a long range interaction in the dark sector usually leading to a fifth force experienced either by standard matter or by Dark Matter (see e.g. Friemann and Gradwohl, 1991, 
Gradwhol and Friemann, 1992, Carroll et al 2009 and 2010). This hypothetical fifth force has been constrained using various astrophysical observations (see Kesden and Kamionkowski, 2006a and 2006b, Carroll et al 2009 and 2010, Stubbs 1993, Bai et al 2015). More recently, a model of Dark Matter coupled to a scalar field has been proposed by Damour et al 1990 . This model leads to a violation of the EEP between Dark Matter and standard matter. Initially introduced as a model leading to a time variation of the gravitational constant $G$, its cosmological implications in the context of late-time cosmology have been thoroughly studied (see Alimi and Füzfa 2007, 2008 and Füzfa and Alimi 2007). In particular, it has been shown that the violation of the EEP in the Dark sector can naturally produce Dark Energy. This violation of the EEP has recently been constrained using galactic observations by Mohapi et al 2016.

The search for a violation of the UFF will be pursued in the future. In particular, the MICROSCOPE satellite, launched successfully on April $25^{\text {th }} 2016$ is expected to test this principle at the level of $10^{-15}$. Several projects aiming at improving current tests have also been proposed.

\section{b) Local Lorentz Invariance}

Local Lorentz Invariance (LLI) is a feature of relativity stating that the outcome of any local nongravitational experiment is independent of the velocity and orientation of the apparatus (see e.g. Will, 2014). While completely integrated into special relativity, the effort to test LLI has recently increased. Indeed, it is often believed that models of quantum gravity will produce violations of Lorentz invariance. These models usually introduce a fundamental length (the Planck length). Since this length is not Lorentz invariant, in most scenarios, a breaking of Lorentz symmetry at some level is produced (see the discussion in Sec. 2.1.2 from Will, 2014 or D. Mattingly, 2005). Several formalisms have been used to test Lorentz symmetry. In the simplest one, one is looking for anisotropies in the speed of light, often parametrized by the $c^{2}$-formalism. This kind of test is usually performed using improved Michelson-like experiments (see e.g. Brillet and Hall, 1979). More recent tests constrain the isotropy of atomic energy levels with a very impressive accuracy (see Lamoreaux et al, 1986).

Another widely used formalism to test Lorentz invariance is called the Robertson-Mansouri-Sexl (RMS) framework (see Robertson 1949 and Mansouri and Sexl, 1977) in which a modification of the kinematic Lorentz transformation is parametrized. Deviations within this formalism are usually searched (see the discussion IV. B. of Turyshev, 2009) using Michelson-Morley experiment (see e.g. Stanwix et al, 2006), Kennedy-Thorndike experiment (see Kennedy and Thorndike, 1932 and Wolf et al 2003) and Ives-Stilwell experiment (see Ives and Stilwell, 1938 and Saathoff et al, 2003). It is interesting to notice that such a test has also been performed using GPS measurements (see Wolf and Petit, 1997). All modern versions of these experiments use atomic spectroscopy, atomic clocks or highly stable oscillators.

The previous formalisms consider mainly the electromagnetic interaction. More recently, a very wide formalism has been developed to consider hypothetical violation of Lorentz symmetry in all fields of physics. This framework has been named Standard Model Extension (SME) and contains an impressive number of parameters encoding hypothetical deviations from the Standard Model 
of particles or from GR (see e.g. Colladay and Kostelecky, 1997, Colladay and Kostelecky 1998, Kostelecky and Mewes, 2005 or Tasson, 2014). A number of these SME coefficients have been constrained by various experiments (for a review of the current constraints on SME parameters, see Kostelecky and Russel, 2011). Let us mention that in this framework, atomic clocks have been successfully used (see e.g. Wolf et al, 2006, Reinhardt et al, 2007, Hohensee et al 2011).

\section{c) Local Position Invariance}

Local Position Invariance (LPI) stipulates that the outcome of any local non-gravitational experiment is independent of the space-time position of the freely-falling reference frame in which it is performed (see e.g. Will, 2014). This principle is mainly tested by two types of experiments: (i) search for variations in the constants of Nature and (ii) redshift tests.

The question of the constancy of the constants of Nature was first addressed by Dirac. This question is driven by the principle of reason: what could be the reasons behind the specific values of the constants of Physics? (see the discussion in Section 2 of Damour, 2012) This argument leads to many developments of extensions of physics where the constants of physics become dynamical entities. In parallel, many observational investigations try and search for any space/time evolution of the constants of Physics (see Uzan, 2011).

Amongst all the observations performed, atomic clocks have an important role leading to currently some of the best constraints currently available. In particular, linear drifts in the evolution of the fine structure constant $\alpha$, in the ratio $\mu$ between the mass of the electron and the mass of the proton and in the ratio between the mass of the light quarks (up and down) and the quantum chromodynamics ( $Q C D$ ) energy scale $\Lambda_{Q C D}$. Several groups in the world have pursued effort to constrain such hypothetical linear drifts: at SYRTE, Observatoire de Paris (see Guéna et al, 2012), NIST (see Rosenband et al 2008), Berkeley (see Leefer et al, 2013), NPL (see Godun et al, 2014), PTB (see Huntemann et al, 2014), ... The current constraints on the variation of the three constants (fine structure constant, ratio between the mass of the electron and the mass of the proton and ration between the mass of the light quarks and the QCD energy scale) are at the level of $10^{-16}$ per year. More recently, searches for a harmonic temporal variation of the constants of Nature using atomic clocks have been performed in Berkeley (see Van Tilburg et al, 2015) and at SYRTE (see Hees et al, 2016a).

In addition to atomic clocks, several astrophysical observations have also been used to search for a temporal evolution of the constants of Nature. In particular, observations of quasar absorption lines at the Very Large Telescope (VLT) in Chile and at the Keck Observatory (Hawaii) lead to observations of $\Delta \alpha / \alpha$ of the order of $10^{-6}$ at cosmological scales, typically for redshifts between 0.2 and 4 (see Webb et al 2003 and Srianand et al 2004). It is interesting to mention that both sets of observations seem to indicate a temporal evolution of the fine structure constant but the two estimations are not compatible with each other. This led the two teams to suggest a dipolar dependence of the fine structure constant in the sky (see Webb et al, 2011). Using a linear interpolation at low redshift, we can compare the order of magnitude of constraints obtained with atomic clocks and using quasar observations. The atomic clock estimations are roughly one 
order of magnitude more accurate than quasar observations. This comparison implies a linear approximation of the cosmological evolution and implies that no screening mechanism is playing a role around the Earth (a screening mechanism can lead to a situation where deviations from GR at cosmological scales are hidden or strongly reduced on local scales, see for example Khoury and Weltman, 2004). Furthermore, observations of the Cosmic Microwave Background (CMB) led to a measurement of $\Delta \alpha / \alpha$ at the level of $10^{-3}$ for a redshift $z \approx 1000$ (see Planck Collaboration, 2013). Finally, analysis of the Big Bang Nucleosynthesis (BBN) also led to an estimation of $\Delta \alpha / \alpha$ at the level of $10^{-3}$ for a redshift $z$ of the order of $10^{10}$ (see Avelino et al, 2001).

In addition to temporal variations of the constants of Nature, one can search for spatial variations. Regarding this, atomic clocks have also been widely used to search for a variation of the constants of Nature with respect to the gravitational potential of the Sun. The idea is to compare two clocks working on different atomic transitions and therefore sensitive differently to the different constants of Physics, located at the same place and search for periodic variations in their frequencies comparison. This kind of test is also known as null redshift experiment (see Will, 2014). Several groups in the world have been measuring this effect which is now constrained at the level of $10^{-6}$ : the SYRTE (see Guéna et al, 2012), the USNO (see Peil et al, 2013), Berkeley (see Leefer et al, 2013), NIST (see Ashby et al 2007), ...

The second way to test the LPI is to measure the gravitational redshift. The gravitational redshift is a consequence of the EEP predicted by A. Einstein, 1911. It was observed for the first time by Pound and Rebka, 1959. A simple and convenient formalism to test the gravitational redshift is to introduce a new parameter $\alpha_{\text {red }}$ defined through (see Will 2014)

$$
\frac{\Delta v}{v}=\left(1+\alpha_{r e d}\right) \frac{\Delta U}{c^{2}}
$$

with $\alpha_{\text {red }}$ vanishing when the EEP is valid. The best constraint on $\alpha_{\text {red }}$ is at the level of $10^{-4}$ and has been obtained in 1976 by comparing the frequency of two clocks: one onboard a rocket and the other one on Earth (see Vessot et al, 1980). Improvements on this test is expected in the near future by using the GNSS satellites Galileo V and VI (see Delva et al, 2015 and section 3 below) and with the Atomic Clock Ensemble in Space project (see Cacciapuoti, 2009 and section 3 below) that aims at comparing atomic clocks on the international space station with atomic clocks on Earth. Finally, a test of the gravitational redshift is expected in the near future using spectroscopy observations of S stars orbiting around our galactic center Sagitarius A* (see Zucker et al, 2006).

\section{d) Summary}

In conclusion, the EEP is an essential part of Einstein's theory that is shared by a number of alternative theories of gravitation. All the theories of gravitation satisfying the EEP are called metric theories. In these theories, it is sufficient to know the space-time metric (to which matter is minimally coupled) to infer all effects produced by gravitation (motion of bodies, light propagation, behavior of clocks, ...). It is interesting to mention that the equivalence principle is a generalization of the fact that all bodies seem to fall with the same acceleration in a gravitational field. Nevertheless, as mentioned by T. Damour (see Damour, 2012): “Despite its 
name, the 'equivalence principle' (EP) is not one of the basic principles of physics. There is nothing taboo about having an observable violation of the EP. In contrast, one can argue (notably on the basis of the central message of Einstein's theory of general relativity) that the historical tendency of physics is to discard any, a priori given, absolute structure (principle of absence of absolute structures)." It is therefore highly important to pursue our quest to test the various facets of this principle. Several on-going projects or proposals aim at improving our current searches for deviations from the EEP. In all the different kind of tests, clocks have an important role regarding tests of the LLI and of the LPI through redshift tests and tests of the constancy of the constants of Nature.

\subsection{Einstein field equations}

The EEP allows one to derive the effects of gravitation from the space-time metric $g_{\mu \nu}$. In order to have a complete theory, one needs to give a prescription to determine the form of this metric. In $\mathrm{GR}$, the form of the metric is determined by solving the Einstein field equations

$$
R_{\mu \nu}-\frac{1}{2} R g_{\mu \nu}+\Lambda g_{\mu \nu}=\frac{8 \pi G}{c^{4}} T_{\mu \nu}
$$

where $R_{\mu \nu}$ and $R$ are the Ricci tensor and scalar curvature from the metric, $G$ the gravitational constant, $c$ the speed of light in vacuum, $T_{\mu \nu}$ the stress-energy tensor and $\Lambda$ the cosmological constant. These equations are making a very interesting link between space-time geometry essentially described in the left part of the equation and the matter/energy content in this spacetime essentially described by the stress-energy tensor. In other words, this set of equations describes how space-time is curved by the presence of matter and energy.

The Einstein field equations can be derived by means of a variational principle from the EinsteinHilbert action (see Einstein, 1916)

$$
S_{\text {grav }}=\frac{c^{4}}{16 \pi G} \int d^{4} x \sqrt{-g}(R-2 \Lambda) .
$$

The total action which describes completely the GR theory is the sum $S_{\text {grav }}+S_{\text {mat }}$ which makes GR an extremely simple theory from a conceptual point of view. It is also interesting to mention that due to the Lovelock theorem, the Einstein field equations are the only second order equations that can be derived from a least-principle action based on the space-time metric (and its derivatives) in a 4 dimensional Riemannian space (see Lovelock 1971, 1972). Following this theorem, alternative (metric) theories of gravitation will always imply one of the following (see Clifton et al, 2012):

- The existence of new fields in addition to (or instead of) the space-time metric

- The existence of higher order derivatives of the metric in the field equations

- To work in a space-time with higher dimension than 4

- To give up locality. 
If the EEP specifies how different types of mass-energy react to gravitation, the Einstein field equations govern how gravitation is generated by matter and energy. A modification of the Einstein-Hilbert action will lead to different field equations that may lead to differences in the space-time metric for a given situation. From an experimental point of view, it is of prime importance to search for metric deviations that would be produced by any deviations from GR. The class of metric theories of gravitation is huge and it is particularly difficult to analyze observational data in a general framework. So far, two frameworks have been widely used to analyze data: the parametrized post-Newtonian (PPN) formalism (see Will, 1993 and 2014) and the fifth force formalism (see Talmadge et al, 1988 and Fischbach and Talmadge, 1999 and Adelberger et al, 2009). As we shall see in the coming sections, these two formalisms have been very precisely constrained with observations at Solar System scales. Nevertheless, it is useful to analyze experimental results in new extended frameworks. Indeed, even if observations lie very close to GR when analyzed within the PPN or fifth force framework, this does not mean that this has to be true in any other framework. The existing frameworks indeed cover a limited set of alternative theories of gravity (see Hees et al, 2012 and 2014a). For example, a formalism aiming at considering violation of Lorentz invariance in the gravity sector has been develop within the SME framework (see Bailey and Kostelecky, 2006). In this formalism, an expansion at the level of the action is performed which naturally leads to a post-Newtonian metric that differs from the PPN one. Other examples of theories not entering the PPN or fifth force frameworks are given by the MOND (MOdified Newtonian Dynamics) phenomenology which produces a quadrupolar deviation from the Newton potential in the Solar System (see Milgrom 2009 and Blanchet and Novak, 2011), by the post-Einsteinian Gravity (PEG, see Jaekel and Reynaud 2005 and 2006), by the parametrized post-Newtonian-Vainshteinian formalism (see Avilez-Lopez et al, 2015), by Horndeski's gravity (see Hohman, 2015), etc ... Currently, only few data analyses are exploring these formalisms or theories.

\section{a) The parametrized post-Newtonian (PPN) formalism}

The PPN formalism is a nice interface between theoretical developments and data analyses. It is fully described in Will, 1993. In this formalism, a phenomenological expansion at the level of the space-time metric is performed by introducing 10 dimensionless parameters. From an observational point of view, these parameters can be constrained regardless of any considerations about the hypothetical underlying theory. From a theoretical point of view, if the post-Newtonian metric of a theory can be matched to the PPN metric, it can automatically be constrained by all observations used to constrain the PPN parameters.

In the simplest case, only two PPN parameters are considered and the space-time metric for a spherically symmetric configuration is given by

$$
d s^{2}=-\left(1+2 \frac{\phi_{N}}{c^{2}}+2 \beta \frac{\phi_{N}^{2}}{c^{4}}+\cdots\right) c^{2} d t^{2}+\left(1-2 \gamma \frac{\phi_{N}}{c^{2}}+\cdots\right) d x^{2}
$$

where $\phi_{N}$ is the Newtonian potential and $\gamma$ and $\beta$ are the PPN parameters. These parameters take the value of 1 in GR and may deviate from 1 in alternative theories. 
The PPN formalism was historically the first one developed and therefore was extensively constrained by several different types of observations. The best constraint on the $\gamma$ parameter was obtained by measuring precisely the Shapiro time delay with the Cassini spacecraft when it was cruising between Jupiter and Saturn in 2003 and is given by (see Bertotti et al, 2003)

$$
\gamma-1=(2.1 \pm 2.3) \times 10^{-5}
$$

This constraint has been confirmed by measuring the light deflection around the Sun using Very Long Baseline Interferometry (see Lambert and Le Poncin-Lafitte, 2011), by using radioscience observations of spacecraft orbiting Mars (see Konopliv et al, 2011) or Mercury (see Verma et al, 2014).

The $\beta$ PPN parameter is essentially constrained through orbital dynamics. Lunar Laser Ranging analysis provides a constraint at the level of $10^{-4}$ (see Williams et al 2009 and 2012). Furthermore, planetary ephemerides analysis currently provides constraints at the level of $10^{-5}$ (see Konopliv et al 2011 for JPL ephemerides, Fienga et al 2015 for the INPOP ephemerides and Pitjeva and Pitjeva, 2013 for the EPM ephemerides).

The other PPN parameters are also very well constrained. An updated list of constraints can be found in Will, 2014. Several projects will improve current constraints in the future like e.g. the GAIA space mission (see Mignard and Klioner, 2010, Mouret 2011 and Hees et al, 2015a) or BepiColombo (see Milani et al, 2002 and Ashby et al, 2007a). Furthermore, several proposals aiming at improving PPN constraints by more than one order of magnitude have been proposed (see section 4 below).

\section{b) The fifth force formalism}

In the fifth force formalism, a Yukawa-type deviation from Newtonian gravity is considered. The deviation from the Newtonian potential is given by

$$
\phi=-\frac{G M}{r}\left(1+\alpha e^{-\frac{r}{\lambda}}\right)
$$

where $\alpha$ is the strength of the interaction and $\lambda$ a characteristic length scale. The idea is to constrain the couple of parameters $(\alpha, \lambda)$. An impressive number of experiments and observations have also been used to constrain this formalism at various scales: at Solar System scales by using planetary ephemerides analyses (see Konopliv et al, 2011), Lunar Laser Ranging observations (see Adelberger et al, 2003), ranging measurements of LAGEOS satellite around Earth (see Fischbach and Talmadge, 1999). At lower distances, several geophysical observations have been used to constrain a hypothetical fifth force. A short distances, torsion balances provide the best constraints. For $\lambda \leq \mu m$, it becomes extremely hard to constrain the strength of the interaction because of several quantum noise sources (like e.g. the Casimir effect). In conclusion, the fifth force formalism is very well constrained except at very low and very large distances where deviations can still be searched for. 


\section{c) The pure gravity sector of the Standard Model Extension}

As mentioned previously, the SME is a very wide framework aiming at considering systematically Lorentz violations in any sector of Physics. In the pure gravity sector, SME can be interpreted as a metric extension of GR. This framework is built by considering an expansion at the level of the action by introducing terms that violate Lorentz symmetry (see Bailey and Kostelecky, 2006). In the minimal SME framework, the resulting post-Newtonian metric is parametrized by a symmetric trace-free tensor $\bar{S}^{\mu \nu}$ (i.e. by 9 coefficients). This metric does not correspond to the PPN metric and therefore, this framework offers new possibilities to search for GR deviations. So far, a couple of postfit data analyses have been performed to constrain the SME coefficients. A postfit analysis means that the analysis has been done in two steps: (i) a standard reduction of the data in GR followed by (ii) a fit of SME signatures in the residuals resulting from the GR reduction. This approach is quite simple to implement in practice but suffers from the fact that all correlations between the SME coefficients and other standard parameters fitted in the analysis is neglected and the obtained uncertainties on the estimated SME parameters are therefore usually underestimated. Therefore, these analyses should be considered as sensitivity analyses only. Sensitivity analyses have been performed with LLR observations (see Battat et al, 2007), atom interferometry measurements (see Müller et al, 2008), binary pulsars observations (see Shao, 2014), planetary ephemerides analyses (see lorio, 2012 and Hees et al, 2015b) and Gravity Probe B analysis (see Bailey et al, 2013).

In addition, very recent analyses of VLBI data and of LLR data have been performed within the SME framework (see Le Poncin-Lafitte et al, 2016, Bourgoin et al. 2016). Contrarily to previous analyses, these studies are not post-fit analyses. Rather, the SME contribution has been added to the full software used to analyze VLBI or LLR data.

\section{d) Modified Newtonian Dynamics (MOND)}

The MOND phenomenology was introduced in 1983 by M. Milgrom (see Milgrom, $1983 \mathrm{a}, \mathrm{b}$ and c) in order to explain observations at the galactic scale (in particular galactic rotation curves) without the introduction of Dark Matter. The main idea behind the MOND phenomenology is to

replace the standard Newton acceleration $g_{\mathrm{N}}$ valid in "high" gravitational field by $\sqrt{a_{0} g_{N}}$ for regions of the Universe where the gravitational field is very low. More precisely, the MOND phenomenology depends on a MOND interpolating function $v$ and on a MOND acceleration scale $a_{0}$. The interpolating function is making the transition between the Newtonian regime and the MONDian regime appearing in low gravitational fields. While developed initially as a purely phenomenological Newtonian model, the MOND phenomenology has later been extended as relativistic theories. A review of different relativistic MOND theories can be found in Bruneton and Esposito-Farèse, 2007 or in Famaey and McGaugh, 2012. A review of the different interpolating functions used in the literature can also be found in Famaey and McGaugh, 2012 (see also Hees et al, 2016b).

Recently, it has been shown that contrarily to what was previously expected, the MOND phenomenology would produce detectable effects in the Solar System. This effect is due to the 
non-linearity of the MOND phenomenology which implies that the external galactic gravitational field will play a role in this theory. This External Field Effect was first discovered by Milgrom, 2010 and was studied later by Blanchet and Novak, 2011. The main contribution from this External Field Effect in the Solar System takes the form of a quadrupolar deviation from the Newton potential

$$
\phi_{N}=-\frac{G M}{r}-\frac{Q_{2}}{2} x^{i} x^{j}\left(e_{i} e_{j}-\frac{1}{3} \delta_{i j}\right)
$$

where the unit vector $e_{i}$ points toward the galactic center and $Q_{2}$ is a parameter that depends directly on the MOND interpolating function $v$ and on the MOND acceleration scale $a_{0}$. Values of this parameter for different interpolating functions are given in Milgrom, 2010, Blanchet and Novak, 2011 and Hees et al, 2016b.

The interesting point is that this modification of gravitation does not enter the fifth force or the PPN formalisms and it can be used efficiently to detect or constrain the MOND phenomenology. A recent analysis of radio tracking data of the Cassini spacecraft when it was orbiting Saturn (between 2004 and 2013) led to a constraint on $Q_{2}$ given by (see Hees et al, 2014b)

$$
Q_{2}=(3 \pm 3) \times 10^{-27} s^{-2}
$$

This constraint on MOND theory is the best currently available on Solar System scales and the propositions to use LISA Pathfinder to detect MOND theory close to a saddle point (see e.g. Bekenstein and Magueijo, 2006) are unlikely to improve this test (see Hees et al, 2016b).

\section{e) Summary}

The Einstein Field Equations determine the form of the metric, or in other words describe how gravitation is generated. They can be derived from the Einstein-Hilbert action which gives GR a very simple form (at least conceptually). Many alternatives theories of gravitation have been developed introducing new additional fields, including higher order derivative terms of the metric in the field equations (like e.g. $f(R)$ gravity), working in higher dimensional space-time, ... These modifications of gravitation are usually motivated by developments of quantum theory of gravity, the unification of the interactions of physics, hypothetical models of Dark Energy and Dark Matter.

From an observational point of view, it is difficult to constrain deviations from GR in a single general framework. Nevertheless, so far, two frameworks have been widely used to search for GR deviations: the PPN formalism and the fifth force framework. As mentioned in this section, a lot of measurements have been used to constrain these, and most of them rely on high performance clocks either directly or indirectly (e.g. through deep space radio ranging). It is therefore interesting to consider other alternative frameworks that allow to search for deviations that may not be seen within the standard formalisms. The gravity sector of SME and the MOND External Field Effect developed above are two examples of these alternative frameworks that have recently been constrained with data but many other examples exist in the literature. 


\subsection{Link between local tests of $G R$ and galactic and cosmological observations}

The previous section was devoted to describe briefly the theoretical basis of GR and how these can be tested experimentally at small scales (Solar System and smaller). Here, we will show how the interplay between local scales and large scales (i.e. galactic and cosmological scales) can be highly beneficious. First of all, a large class of alternative theories of gravitation have been developed to specifically tackle the Dark Matter and/or Dark Energy problematics. The idea is to modify the gravitation theory to explain observations that require the introduction of Dark Matter and/or Dark Energy without adding new components to the standard model of particles or introducing the cosmological constant. An excellent review of a large class of theories developed for cosmological purposes can be found in Clifton et al, 2012. As can be seen from this review (and the list is not exhaustive), the number of theoretical models developed is huge. It is therefore of prime importance to confront these GR extensions intended for large scales, with small scale observations to try and identify the theories that are viable on all scales in our Universe. The idea is therefore to combine observations at different scales to reduce as much as possible the compatible parameters space. In the next section, we will develop several examples to illustrate this point and to highlight the power of this type of combined analysis. Ultimately, one would try to identify a theory (or set of theories) that satisfies all current observations at all scales and that will solve some (or all if we are optimistic) problems related to the gravitation theory.

\section{a) "Dilaton-like" scalar fields}

Amongst the zoo of alternative theories of gravitation, tensor-scalar theories are very popular and have been studied in great details. The pioneering work on tensor-scalar theories was performed by Jordan, Brans and Dicke (see Brans and Dicke, 1961) motivated by considerations about Mach's principle. From there, an impressive number of studies of tensor-scalar theories have been performed by modifying the interaction with the space-time metric, modifying the kinetic part of the scalar field action, by considering different type of potentials, by allowing more than second order derivatives in the field equations, by considering several different couplings to matter, and so on... The motivations to consider such models are also very wide: they are considered as phenomenological model inspired by unification theory (see e.g. Damour and Polyakov, 1994), as higher dimensional theoretical models (see e.g. Overduin and Wesson, 1997), as a model of Dark Matter (see e.g. Stadnik and Flambaum, 2014 or Arvanitaki et al, 2015), as model of Dark Energy (see for example Khoury and Weltman, 2004),...

The dilaton theories are a class of models where the scalar field is non-minimally coupled to matter, therefore breaking explicitly the EEP. As an example, the coupling between matter and the scalar field can be parametrized by the interaction Lagrangian (see e.g. Damour and Donoghue, 2010)

$$
L_{i n t}=\varphi\left(\frac{d_{e}}{4 \mu_{0}} F_{\mu \nu} F^{\mu \nu}-\frac{d_{g} \beta_{g}}{2 g_{3}} G_{\mu \nu}^{A} G_{A}^{\mu \nu}-c^{2} \sum_{i=e, u, d}\left(d_{m_{i}}+\gamma_{m_{i}} d_{g}\right) m_{i} \overline{\psi_{i}} \psi_{i}\right),
$$


where $F_{\mu \nu}$ is the standard electromagnetic Faraday tensor, $\mu_{0}$ the vacuum magnetic permeability, $G_{\mu \nu}^{A}$ the gauge invariant gluon strength tensor, $g_{3}$ the QCD gauge coupling, $\beta_{3}$ denotes the $\beta$ function for the running of $g_{3}, m_{i}$ the mass of the fermions ( $\mathrm{i}=\mathrm{e}, \mathrm{u}, \mathrm{d}$ for electron and up/down quark), $\gamma_{m_{i}}$ the anomalous dimension giving the energy running of the masses of the QCDcoupled fermions and $\psi_{i}$ the fermions spinor. This Lagrangian is parametrized by five dimensionless coefficients $d_{e}, d_{m_{e}}, d_{m_{u}}, d_{m_{d}}$ and $d_{g}$ that characterize the coupling between the scalar and standard model fields. More complex type of couplings or coupling to other part of the standard model (e.g. to gauge bosons of the weak interaction) have also been explored (see e.g. Stadnik and Flambaum, 2014 and references therein). It is well known that such a model will induce a violation of the Einstein Equivalence Principle for baryonic matter (see e.g. Damour and Donoghue, 2010) and can be constrained using all observations related to the EEP (see section 2.1 above).

For example, in the case where the action of the scalar field is standard and no potential is considered (massless field), it is known that UFF tests constrain the $d_{g}, d_{m_{u}}$ and $d_{m_{d}}$ coefficients pretty well (see Damour and Donoghue, 2010) while tests with clocks (redshift tests or tests of the constancy of the constants of Physics) can be interesting regarding the $d_{e}$ and more importantly the $d_{m_{e}}$ coefficients (see Hees and Minazzoli, 2015). In the case where the scalar field is slightly massive, clock comparisons can be much better than UFF experiments (see Van Tilburg et al, 2015 or Hees et al, 2016a). This illustrates the complementarity of the different types of experiments. This class of theories has several links to galactic and cosmological scales. First of all, the dilaton coupling introduced in the interaction Lagrangian above has very specific signatures at the cosmological level and can impact several cosmological observations (Cosmic Microwave Background - $\mathrm{CMB}$ - temperature, distance duality relation, ...). Furthermore, this class of models has been studied as Dark Matter and Dark Energy model and it is therefore interesting to make a combined analysis to constrain these models. We will explore these three cases here in order to illustrate the strong interaction between the different scales.

\section{i. Some cosmological observables}

In this section, we will consider a dilatonic field that couples only to EM so that the related part of the action is

$$
S_{E M}=-\frac{1}{4 \mu_{0} c} \int d^{4} x \sqrt{-g}\left(1-d_{e} \varphi\right) F_{\mu \nu} F^{\mu \nu}+q_{p} \int A_{\mu} d x^{\mu}
$$

where $q_{p}$ is the electric charge of a particle interacting with the EM field. It is important to point out that in order to preserve the $U(1)$ gauge invariance, the interaction part of the electromagnetic Lagrangian cannot include a contribution from the scalar field (see Bekenstein, 1982 and Hees et al, 2015c). The coupling introduced breaks explicitly the EEP (in particular, it cannot be absorbed through a conformal transformation). As mentioned previously, this kind of coupling has implications on the universality of free fall and on variations of fundamental constants. In addition to these effects, four cosmological observables are modified (with respect to GR) and are intimately related to each other in this class of theories (see Hees et al, 2014c): (i) temporal variation of the fine structure constant, (ii) violation of the distance-duality relation, 
(iii) modification of the evolution of the $\mathrm{CMB}$ temperature and (iv) $\mathrm{CMB}$ spectral distortions. The theoretical derivation can be found in (Hees et al, 2014c). It is worth to insist on the fact that the derivation relies only on the matter part of the action and not on the gravitational part. This means that our results apply to a very wide class of gravitation theories. In a Friedman-LemaîtreRobertson-Walker space-time, the expressions of the four observables are given by the following expressions:

- Temporal variation of the fine structure constant. A straightforward identification in the action leads to (see e,g, Uzan, 2011) $\frac{\Delta \alpha}{\alpha}=\frac{\alpha(z)-\alpha_{0}}{\alpha_{0}}=d_{e}\left(\varphi(z)-\varphi_{0}\right)$, where $z$ is the redshift and subscripts 0 refer to $z=0$.

- violation of the cosmic distance-duality relation. The optic geometric limit of the modified Maxwell equations shows that photons propagate on null geodesics but their number is not conserved due to an exchange with the scalar field (see also Minazzoli and Hees, 2014). Therefore, the expression of the angular diameter distance $\left(D_{A}\right)$ is the same as in GR but this leads to a modification of the distance-luminosity expression $\left(D_{L}\right)$ (see also Minazzoli and Hees, 2014) and hence to a violation of the cosmic distance-duality relation:

$$
\eta(z)=\frac{D_{L}(z)}{\left[D_{A}(z)(1+z)^{2}\right]}=1+\frac{d_{e}}{2}\left(\varphi(z)-\varphi_{0}\right)
$$

- modification of the evolution of the $\mathrm{CMB}$ temperature. Considering the $\mathrm{CMB}$ as a gaz of photons described by a distribution function solution of a relativistic Boltzman equation and using the geometric optic approximation of the modified Maxwell equations leads to a modification of the $\mathrm{CMB}$ temperature evolution:

$$
T(z)=T_{0}(1+z)\left[1+0.12 d_{e}\left(\varphi(z)-\varphi_{0}\right)\right]
$$

- spectral distortion of the $\mathrm{CMB}$. Using the same approach as the one sketched in the last item, one can show that the evolution of the $\mathrm{CMB}$ radiation leads to deviations from its black body spectrum. When this deviation is parametrized by a chemical potential $\mu$, one can show that its expression at current epoch is given by $\mu=0.47 d_{e}\left(\varphi\left(z=z_{C M B}\right)-\right.$ $\left.\varphi_{0}\right)$.

To summarize, a coupling between the scalar field and EM implies that the four observables are intimately linked to each other through the relations

$$
\begin{aligned}
& d_{e}\left(\varphi(z)-\varphi_{0}\right)=\frac{\Delta \alpha}{\alpha}=2(\eta(z)-1)=8.33\left(\frac{T(z)}{T_{0}(1+z)}-1\right) \mu \\
& \quad=0.47 \frac{\Delta \alpha\left(z_{C M B}\right)}{\alpha}=0.94\left(\eta\left(z_{C M B}\right)-1\right)=3.92\left(\frac{T\left(z_{C M B}\right)}{T_{0}\left(1+z_{C M B}\right)}-1\right) .
\end{aligned}
$$

These relations hold for all theories whose matter part of the action can be cast in the form of the action (12). This class of theories is very large and includes all metric theories. These relations imply also that if a deviation of the EEP is observed around Earth, it is likely to have cosmological counterparts as well, which would be an excellent check to confirm any observed deviations.

These relations can also be used for two distinct purposes: (i) use measurements of one type of observations to constrain another type (for example, use observation of the variation of $\alpha$ to constrain the evolution of $T_{C M B}$ ) and (ii) use two different types of observations and see if they are consistent with the relations above to test them. 
From an observational point of view, several parametrizations of the distance-duality relationship have been considered in the literature: $\eta(z)=1+\eta_{0}, \eta(z)=1+\eta_{1} z, \eta(z)=$ $1+\eta_{2} \frac{z}{1+z}, \eta(z)=1+\eta_{3} \ln (1+z), \quad \eta(z)=(1+z)^{\varepsilon}$. On the other hand, essentially one parametrization has been used to search for deviations in the evolution of the CMB temperature $T(z)=(1+z)^{1-\beta}$. Assuming that the theory of gravitation is described by the multiplicative coupling introduced in Eq (12) (which is a large class of theories including GR), we can use the relations from Eq. (13) to transform observational constraints on one type of observations into constraints on another type. As an example, we use three datasets of $\alpha$ measurements: precise laboratory clock measurements of variations of $\alpha$ providing the constraint (see Rosenband et al, 2008) $\dot{\alpha} / \alpha=(-1.6 \pm 2.3) \times 10^{-17} / y r, 154$ quasar absorption lines observed at the VLT (see King et al, 2012) and 128 quasar absorption lines observed at the Keck observatory (see Murphy et al, 2003). Using separately these three datasets and Eq (13), we constrain the parameters $\eta_{i}$, $\varepsilon$ and $\beta$ by using a Bayesian inversion (see Hees et al, 2014c).

\begin{tabular}{cccc}
\hline Parameter & \multicolumn{3}{c}{ Estimation $\left[\times 10^{-7}\right]$} \\
& VLT & Keck & Clocks \\
\hline$\eta_{0}$ & $10 \pm 6$ & $-29 \pm 10$ & - \\
$\eta_{1}$ & $8.4 \pm 3.5$ & $-16 \pm 6$ & $1.0 \pm 1.4$ \\
$\eta_{2}$ & $20 \pm 10$ & $-49 \pm 17$ & $1.0 \pm 1.4$ \\
$\eta_{3}$ & $14 \pm 6$ & $-30 \pm 11$ & $1.0 \pm 1.4$ \\
$\varepsilon$ & $14 \pm 6$ & $-30 \pm 11$ & $1.0 \pm 1.4$ \\
\hline$\beta$ & $-3.3 \pm 1.5$ & $7.2 \pm 2.5$ & $-0.3 \pm 0.3$ \\
\hline
\end{tabular}

The constraints derived from clocks measurements rely only on one observation and is valid only if no screening mechanism occurs around Earth. The obtained constraints improve by 5 orders of magnitude direct observations of $\eta$ or of the $\mathrm{CMB}$ temperature. A similar analysis has been performed in order to test the relations given by Eq. (13). No deviations have been observed at the level of $10 \%$. The main limitation in this test is currently coming from angular diameter observations. The future project EUCLID or the SKA are expected to improve these by one order of magnitude.

\section{ii. Dilaton field as Dark Matter}

Another example of interaction between laboratory and larger scales is to consider models where Dark Matter is made of a non-minimally coupled scalar field. While DM particles have been widely searched with high energy physics measurements, gravitational observations can also provide some insight in this search. For example, a model of scalar DM has been proposed (see e.g. Derevianko and Pospelov, 2014, Stadnik and Flambaum, 2015a and b, Arvanitaki et al, 2015)

$$
S=\frac{c^{3}}{16 \pi G} \int d^{4} x \sqrt{-g}\left[R-2 g^{\mu \nu} \partial_{\mu} \varphi \partial_{\nu} \varphi-2 \frac{c^{2}}{\hbar^{2}} m_{\varphi}^{2} \varphi^{2}\right]+S_{S M}\left[g_{\mu \nu}, \psi\right]+S_{i n t}\left[g_{\mu \nu}, \varphi, \psi\right]
$$

where $S_{S M}$ represents the Standard Model action and $S_{\text {int }}$ is the action of the interaction between the scalar field and matter constructed from the Lagrangian given by Eq. (11). This class 
of models has several gravitational signatures that can be searched for at all scales of physics (see e.g. Arvanitaki et al, 2015 and Stadnik and Flambaum, 2015a). In particular, one can show that under reasonable hypotheses, the cosmological evolution of the scalar field is harmonic: $\varphi=$ $c s t+\varphi_{0}\left[\cos c^{2} m_{\varphi} t / \hbar+\delta\right]$. For low $m_{\varphi}$ the mean cosmological pressure due to the harmonic part of the scalar field vanishes allowing to identify the scalar field as DM. This identification implies that the amplitude of the scalar field oscillation $\varphi_{0}$ is directly related to the DM energy density, which is observed in our galaxy as being roughly $0.4 \mathrm{GeV} / \mathrm{cm}^{3}$ (see McMillan, 2011). Such a model would produce oscillations in the different constants of Physics that can be searched for in comparison of atomic clocks frequency (see Arvanitaki et al, 2015 and Stadnik and Flambaum, 2015a). These oscillations depend directly on the matter/scalar field coupling constants $d_{i}$ introduced in the Lagrangian from Eq. (11). Recently, this model has been constrained by using Dysprosium (Dy) atomic transitions at Berkeley (see Van Tilburg et al, 2015) and using Cs/Rb atomic transitions at SYRTE (see Hees et al, 2016a). The best constraint on the $d_{i}$ coefficients obtained using atomic clocks is shown in Figure 1. We can exclude couplings larger than $5.3 \times$ $10^{-4}$ at any $m_{\varphi}$ within our range, with our most stringent limit being as low as $3.8 \times 10^{-9}$ at $m_{\varphi}=1.4 \times 10^{-23} \mathrm{eV} / \mathrm{c}^{2}$. Although, these constraints are numerically much better than the ones obtained with UFF experiments (see Arvanitaki et al, 2016), they are complementary to those of Van Tilburg et al, 2015 and also to those coming from tests of the UFF as they probe different combinations of the coupling constants $d_{i}$.

This example shows once again how local measurement can help to constrain models constructed in order to explain large scales observations.

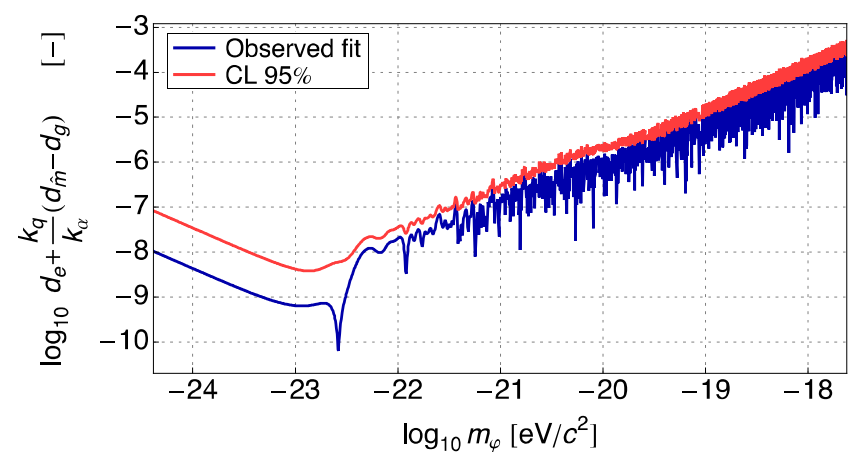

Figure 1: Estimated values of the linear combination $d_{e}+0.043\left(d_{\hat{m}}-g_{g}\right)$ of coupling constants $d_{i}$ between a massive scalar field and standard matter fields as a function of scalar field mass $m_{\varphi}$. The best fit values are shown in blue, with the $95 \%$ confidence upper bounds in red. From Hees et al, 2016a.

\section{iii. Dilaton field as Dark Energy}

The same action as the one introduced in the previous section, but with a vanishing potential, has also been studied in the context of Dark Energy - DE (see e.g. Carroll, 1998 or Nunes and Lidsey, 2004 and references therein). In this case, it is useful to work with the cosmological energy density and pressure associated with the scalar field and it can be shown that the DE equation of state $w_{\varphi}=p_{\varphi} / \rho_{\varphi}$ is given by (see Nunes and Lidsey, 2004) $w_{\varphi}=-1+\frac{2}{3} \frac{\varphi^{\prime 2}}{\Omega_{\varphi}}$ where $\Omega_{\varphi}=\frac{8 \pi G \rho_{\varphi}}{3 H^{2} c^{2}}$. 
This equation allows to derive an expression that makes the connection between the temporal evolution of $\alpha$ to cosmological variables (see e.g. Martins and Pinho, 2015)

$$
\left.\frac{\dot{\alpha}}{\alpha}\right|_{0}=-d_{e} H_{0} \sqrt{\frac{3}{2} \Omega_{\varphi 0}\left(1+w_{\varphi 0}\right)} .
$$

This relation is very useful in order to combine data in a global analysis. For example, Martins and Pinho, 2015 uses atomic clocks constraints on $\dot{\alpha} / \alpha$ and cosmological observations (Supernovae la and Hubble parameter measurements) in order to constrain $d_{e}$ and $w_{\varphi 0}$ (under the assumption that $w_{\varphi}$ is constant and using a fixed value of the Hubble constant of $70 \mathrm{~km} / \mathrm{s} / \mathrm{Mpc}$ ). Figure 2 represents the constraints in the $\left(d_{e}, w_{\varphi 0}\right)$ plane obtained using atomic clock observations only, using cosmological observations only and using a combination of both datasets. It is interesting to notice that both individual datasets are sensitive to only one of the parameters. It is really the combination of local measurements with large scales observations that seriously constrains this model.

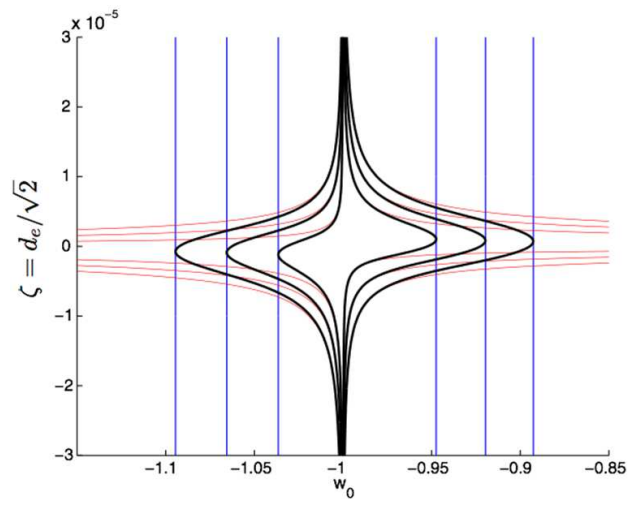

Figure 2 : One-, two-, three- sigma constraints on the $d_{e}-w_{0}$ plane obtained by using atomic clock measurements (only red curves), cosmological data only (blue lines) and a combination of both datasets (black curves). From Martins and Pinho, 2015.

\section{b) The chameleon field}

The examples developed in the previous section are all based on a dilaton-like model, meaning that they all break the EEP. The interest of combining small scale measurements with large scales observations is of course not restricted to this class of theories. As an example, in this section, we will show that the chameleon fields are also interesting in this perspective.

The chameleon fields have been introduced by Khoury and Weltman, 2004a and $b$ and are the first example of what is nowadays known as "screening mechanism". A screening mechanism refers to a mechanism that hides a large deviation from GR in certain regions of space-time. The main motivation behind these mechanisms is coming from the DM and DE problematic and trying to solve them by modifying significantly the GR theory at large scales. In order to satisfy the stringent local tests of gravitation, a screening mechanism is often involved. In the particular case of the chameleon fields, the screening is based on the local matter density: in regions of space time where the matter density is large (like e.g. in the Solar System), the chameleon field is highly 
massive and therefore frozen and undetectable while in region of space-time where the matter density is low (i.e. at cosmological scales), the chameleon field is nearly massless and can eventually play the role of $\mathrm{DE}$. The original chameleon model can be expressed as

$$
S=\int d^{4} x \sqrt{-g}\left[\frac{m_{p}^{2}}{16 \pi} R-\frac{1}{2} \partial_{\mu} \phi \partial^{\mu} \phi-V(\phi)\right]+S_{m a t}\left[\tilde{g}_{\mu \nu}=A^{2}(\phi) g_{\mu \nu}, \psi\right]
$$

where $m_{p}^{2}=1 / G$ (here, we use natural units in which $\hbar=c=1$ ) and $A(\phi)$ is a coupling function here chosen to be $A(\phi)=e^{k \phi / m_{p}}=e^{\phi / M}$. The potential $V(\phi)$ needs to be a runaway potential. Originally, a Ratra-Peebles potential parametrized by 2 constants $\alpha$ and $\Lambda$ was considered $V(\phi)=\frac{\Lambda^{4+\alpha}}{\phi^{\alpha}}$ (see Ratra and Peebles, 1988). It can be shown that the evolution of the scalar field is driven by an effective potential that depends on the local matter density, which is the seed of the screening mechanism.

Since this class of theory is built in order to make a link between local scales and large scales, it is interesting to combine local measurements with cosmological observations. For example, in Hees and Füzfa, 2012, Supernovae la observations are combined with the $\gamma$ PPN measurement in order to constrain the parameters of this theory. Figure 3 shows the constraints obtained using the two types of observations. The cosmological constraint is nearly insensitive to the value of the coupling parameter $k$ and is therefore very similar to the constraint obtained for quintessence fields. On the other hand, the Cassini constraint is very sensitive to the value of the parameter $k$. As can be seen in this Figure, the original chameleon theory is not able to explain the cosmological observations while satisfying the local tests of gravitation. A further detailed analysis (see Hees and Füzfa, 2012) shows that for parameters that can explained the Supernovae la observations, the screening mechanism is not at work in the Solar System, therefore producing unacceptable deviations. This data analysis was confirmed by analytical calculations in Wang et al, 2012.

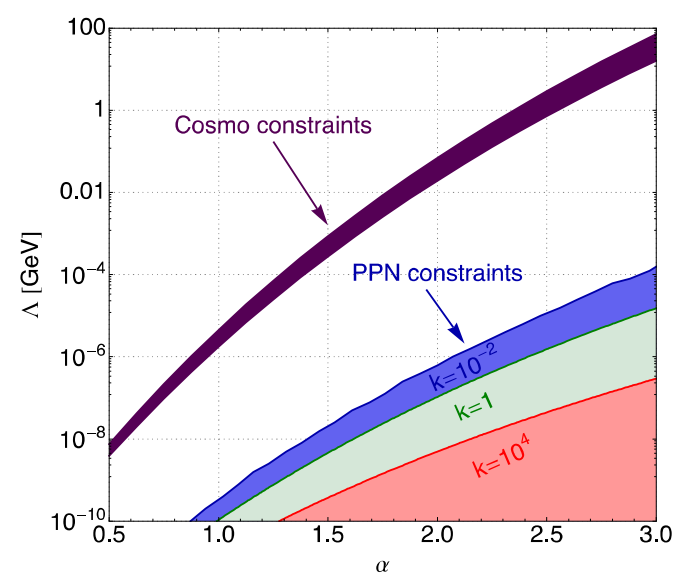

Figure 3 : Representation of the constraints in the $(\alpha, \Lambda)$ plane obtained with Supernovae la observations and with the Cassini PPN constraints (see Eq. (0) above). The cosmological constraint is nearly insensitive to the value of $k$ while the PPN constraint depends on the value of the coupling parameter. From Hees and Füzfa, 2015. 
One solution to save the chameleon theory is to consider a more complex potential like e.g. $V(\phi)=$ $\Lambda^{4} e^{\Lambda^{\alpha} / \phi^{\alpha}} \approx \Lambda^{4}+\frac{\Lambda^{4+\alpha}}{\phi^{\alpha}}$ (see e.g. Zlatev et al, 1999) which from a practical point of view corresponds to adding a cosmological constant to the standard Ratra-Peebles potential. In this case, the first term is responsible for DE while the Ratra-Peebles potential is responsible for the screening mechanism. This model is able to drive the cosmic acceleration observed today if $\Lambda \approx$ $2.4 \mathrm{meV}$ (see Hamilton et al, 2015), leaving the $k$ parameter nearly unconstrained. It is interesting to notice that in this case, atom interferometry measurements can provide a very stringent constraint. Indeed, it has been proposed by Burrage et al, 2015 to use atom interferometry in a large vacuum chamber. The idea is to screen the scalar field generated outside the chamber and to look for a chameleon field generated by atoms inside the chamber. Since atoms are very small, these chameleon fields will not be screened and can therefore be detected. This measurement has been performed in Berkeley (see Hamilton et al, 2015) and excludes an impressive part of the parameter space as can be seen from Figure 4.

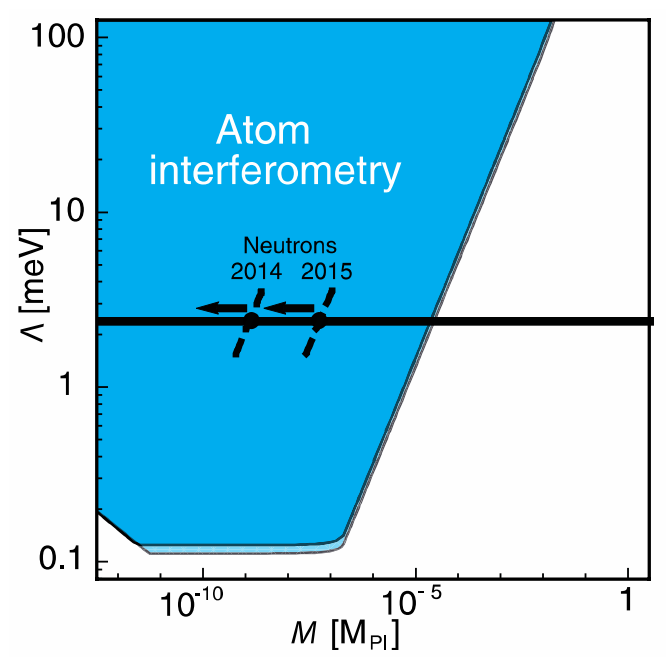

Figure 4: 95\% confidence region of exclusion of the chameleon parameters from atom interferometry for $\alpha=1$. The light blue area corresponds to another modeling of the vacuum chamber showing the robustness of the results. The horizontal thick line represents the value $\Lambda=2.4 \mathrm{meV}$ where the chameleon field would be able to drive the cosmic acceleration. From Hamilton et al, 2015.

In conclusion, the chameleon field is also an interesting theory where the combination of data from local scales and larger scales is highly fruitful. This theory is not ruled out per se but the open parameter space becomes very narrow. The question of fine-tuning can therefore be raised.

c) Modified Newtonian Dynamics (MOND)

The last example of a theory that benefits from a combined analysis of local scale measurements with larger scales observations is the MOND phenomenology. As already mentioned in Section 2.2 above, this phenomenology depends on an interpolating function $v$ making the transition between the Newtonian gravity and the MONDian gravity and on a MOND acceleration scale $a_{0}$. 
Different functions $v$ have been considered in the literature (see e.g. Famaey and McGaugh, 2012) in order to explain galactic observations (like e.g. rotation curves). As developed in Section 2.2 above, it has been recently shown that this phenomenology produces an effect in the Solar System: the External Field Effect. This effect is parametrized by a quadrupolar moment $Q_{2}$ that depends on the interpolating function $v$ and on the acceleration scale $a_{0}$. This quadrupolar moment has been constrained using Cassini radioscience tracking measurements (see Hees et al, $2014 \mathrm{~b}$ and Eq. (00) above). Therefore, this theory will also benefit from a combined analysis including Solar System measurements and galactic rotation curve observations. This analysis is presented in Hees et al, 2016b. The idea is to use observations of 27 galactic rotation curves (using data from Swaters et al, 2010) and to fit them with different MOND phenomenology. In this analysis, 31 different interpolating functions $v$ have been considered. For each of these interpolating functions, an optimal value of the MOND acceleration $a_{0}$ scale has been determined. This value leads to a prediction for the MOND quadrupolar moment $Q_{2}$ that arises in the Solar System. It is then possible to compare this value with the Cassini constraint (see Eq. (10) above). Out of the 31 functions considered, only 10 (if one choses a very conservative approach) are able to explain the rotation curves and the Solar System observations. It is worth mentioning that the inclusion of the last years of Cassini tracking measurements (the analysis from Hees et al, 2014c includes observations up to 2013 only) further improves the constraint on $Q_{2}$ (W. Folkner, 2016, private communication) and will reduce the number of viable MOND interpolating functions. This shows once again the interest of combining observations from different scales.

\section{d) Summary}

In this section, we have presented a few examples where the combination of measurement at local scales (in the lab or at the scale of the Solar System) with larger scales observations (either galactic observations or cosmological observations) leads to very fruitful results. Indeed, the modifications of the gravitation theory made in order to explain galactic or cosmological observations may produce detectable effects at smaller scales (sometimes unexpected). It is therefore highly interesting to combine all these observations in order to facilitate a detection of a deviation from GR or to produce the most stringent constraints within the zoo of alternative theories of gravitation. The examples sketched in this section show the power of such combined analysis and gives strong motivation to pursue these.

\subsection{Conclusion}

In this section we have provided some of the theoretical background that provides the motivation for experimental tests of gravitation, and is generally used to evaluate and compare such tests. Since the advent of general relativity, gravitation is intimately related to space-time, and the most accurate space-time metrology is based on atomic clocks and the exchange of electromagnetic signals. It is thus no surprise that many of the best tests of gravitation are based on high accuracy atomic clocks on ground and in space. This will be further illustrated by some specific examples in the next section. 


\section{Some past, present and near future experiments}

In this section we describe four experiments in fundamental physics that rely on clocks and timing measurements. Three of them concern a test of the Einstein Equivalence Principle via the gravitational redshift (Local Position Invariance) as described in section 2.1. The last one is a test of the Einstein field equations in the parametrized post Newtonian (PPN) framework described in section 2.2. Those experiments have either provided the most stringent constraints in their respective domain to date, or are expected to do so in the very near future (next few years), and are in that sense key experiments in the domain of fundamental physics.

\subsection{Gravity probe A (GP-A)}

The most precise test of the gravitational redshift to date has been realized with the VessotLevine rocket experiment in 1976, also named the Gravity Probe A (GP-A) (Vessot 1979, 1980, 1989). The goal of this experiment was to perform a gravitational redshift test by monitoring the variation of an onboard hydrogen-maser (H-maser) when compared to ground $\mathrm{H}$-masers.

\section{a) Description of the experiment}

On June 18,1976 , a Scout D rocket was launched on a parabolic trajectory from the Wallops island, Virginia, carrying an atomic $\mathrm{H}$-maser clock as the payload. The frequency of signals from the onboard clock was monitored by two $\mathrm{H}$-masers on the ground at Merrit Island, Florida. The first-order Doppler shift in the signals was eliminated by a two-way transponder link to the payload. We show on figure 5 the approximate trajectory of GP-A in inertial space. It was

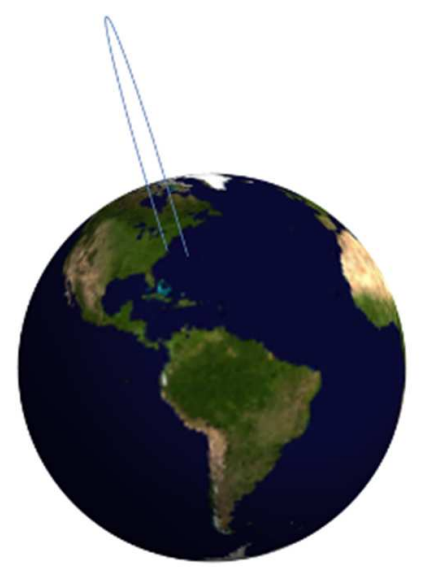

Figure 5: Approximate trajectory of GP-A.

launched at 11:41 GMT and the impact was at $13: 37 \mathrm{GMT}$ at latitude $29^{\circ} 44 \mathrm{~N}$, longitude $47^{\circ} 75$ $\mathrm{W}$. The experiment was therefore completed after a total time of $1 \mathrm{~h} 58 \mathrm{mn}$. However the usable 
data spans over around $1 \mathrm{~h} 20 \mathrm{~min}$.

The main parts of the payload consisted of a H-maser clock, an S-band transmitter phase-locked to the maser, and an S-band transponder (see figure 6). The payload weighed $57 \mathrm{~kg}$ and was spin stabilized to maintain its orientation in inertial space. The S-band signals were received and transmitted from a common antenna located on the spin axis at the aft end of the payload. The principle of the Doppler-cancelling system is shown on figure 6: the uplink signal 1 is phase-locked to the ground $\mathrm{H}$-maser, sent to the onboard transponder and sent back to the ground, which gives a beat frequency $\left(f_{0}\right)^{\prime \prime}-f_{0}$. The downlink signal 3 , phase-locked to the onboard H-maser, is mixed with the ground $\mathrm{H}$-maser frequency and gives a beat frequency $\left(f_{0}\right)^{\prime}-f_{0}$. Half of the beat frequency $\left(f_{0}\right)^{\prime \prime}-f_{0}$ is subtracted from the beat frequency $\left(f_{0}\right)^{\prime}-f_{0}$, thus cancelling the first-order Doppler effect. Moreover, the frequencies of the three signals are chosen such that the first-order ionospheric delay is canceled. The remaining error coming from the ionosphere is below $5 \times 10^{-15}$ in relative frequency.

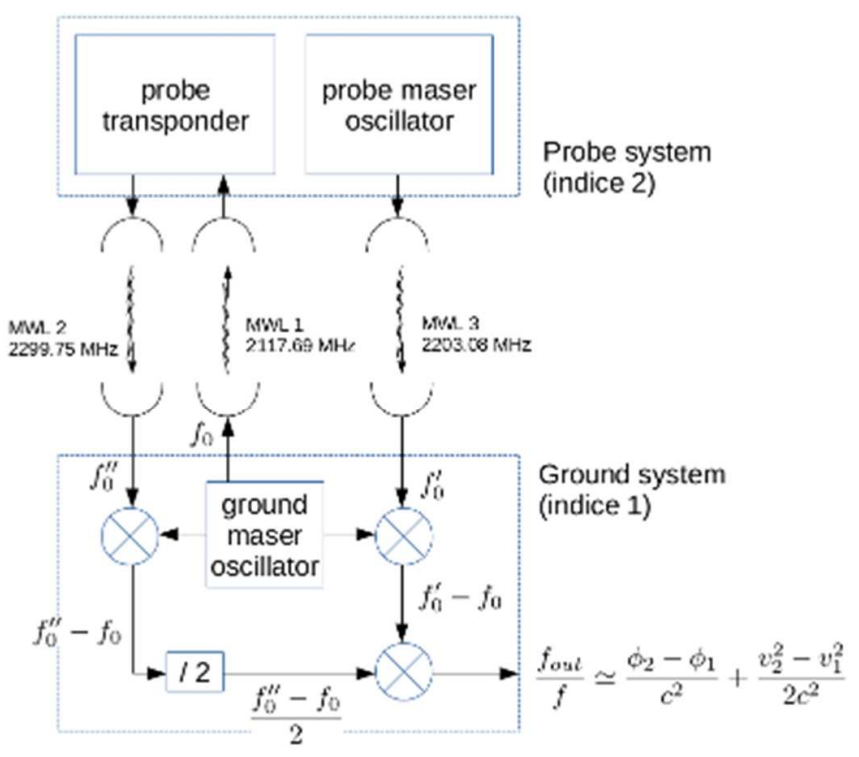

Figure 6: Schematic concept of the Doppler-cancelling system

b) Predicted frequency shift

The proper time to coordinate time ratio of an ideal clock can be written in a quasi-inertial coordinate system centered on Earth as:

$$
y \equiv \frac{d \tau}{d t}=1-\frac{G M}{r c^{2}}-\frac{v^{2}}{2 c^{2}}
$$


where $G M$ is the gravitational parameter of the Earth, $c$ is the velocity of light, $r$ is the distance of the clock from the Earth center, and $v$ is the coordinate velocity of the clock. The two small terms at the r.h.s. of the equation are usually called gravitational redshift and second order Doppler effect.

We show on figure 7 the predicted frequency shift during the GP-A experiment based on an approximated trajectory. We can see that the variation of the second order Doppler effect is almost the same as the gravitational redshift. This is generally the case for an almost Keplerian orbit, because the reduced energy $E=v^{2} / 2-G M / r$ is conserved. Therefore we get for an almost Keplerian orbit:

$$
\delta\left(\frac{v^{2}}{2 c^{2}}\right) \simeq \delta\left(\frac{G M}{r c^{2}}\right)
$$

where $\delta()$ is a small variation with respect to time. This is the case for GP-A because the trajectory is quasi-keplerian. It is almost a parabola as it has a very high eccentricity ( $\simeq 0.999)$.

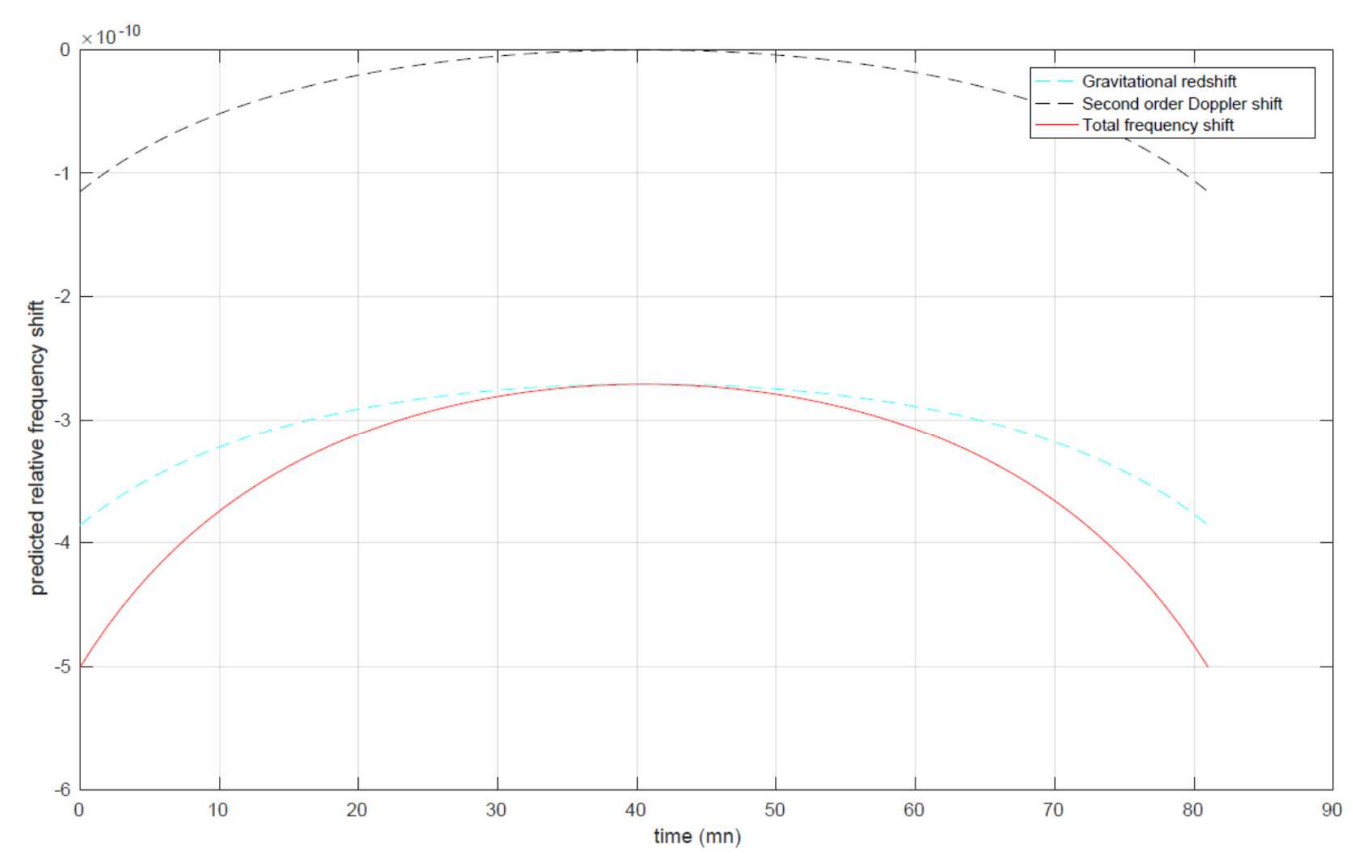

Figure 7: Predicted relative frequency shift for the GP-A experiment, based on an approximated trajectory. 


\section{c) Error budget}

We reproduce the error budget of GP-A from (Vessot 1989) in table 1 below. The combined random and systematic errors sum up to $15.5 \times 10^{-15}$, whereas the variation of gravitational redshift over useful data is estimated to be $1.1 \times 10^{-10}$, which can be seen on figure 7 . Therefore the estimated accuracy on the LPI violation is $1.4 \times 10^{-4}$.

\begin{tabular}{|l|c|c|}
\hline & $\begin{array}{c}\text { GP-A } \\
(2 \text { hours })\end{array}$ & $\begin{array}{c}\text { orbital test } \\
(18-24 \text { hours })\end{array}$ \\
\hline random noise & & \\
Overall microwave system noise & $13.0 \times 10^{-15}$ & $\lesssim 10^{-16}$ \\
including maser instability & $2.5 \times 10^{-15}$ & $2.5 \times 10^{-15}$ \\
Uncancelled atmospheric noise & $5.0 \times 10^{-15}$ & $0.2 \times 10^{-15}$ \\
Uncancelled ionospheric noise & $3.0 \times 10^{-15}$ & $1.0 \times 10^{-15}$ \\
\hline Systematic errors & $4.5 \times 10^{-15}$ & $1.0 \times 10^{-15}$ \\
Ground clock & & \\
Space clock & $3.3 \times 10^{-15}$ & $1.0 \times 10^{-15}$ \\
Errors coming from position & $15.5 \times 10^{-15}$ & $4.7 \times 10^{-15}$ \\
and velocity uncertainties & $1.1 \times 10^{-10}$ & $4.8 \times 10^{-10}$ \\
\hline Combined random and systematic errors & $141 \times 10^{-6}$ & $9.9 \times 10^{-6}$ \\
\hline Variation of gravitational redshift over useful data \\
Predicted error in a single redshift measurement
\end{tabular}

Table 1: Error budget of the GP-A experiment assuming a total time of around 2 hours, and of a similar experiment in orbit assuming a 18-24 hours orbital period (reproduced from Vessot 1989)

The limiting error comes from the overall microwave system noise and maser instability. That is why Vessot (1989) proposed to perform a similar experiment in orbit. Averaging over a longer time, the system noise is below $10^{-16}$. Vessot evaluated the random noise to be $3.2 \times 10^{-15}$ for one orbit. Averaging over 30 orbits, the random error becomes $3.2 \times 10^{-15} / \sqrt{30} \simeq 5.8 \times 10^{-16}$ . Assuming a contribution of systematic errors of $1.7 \times 10^{-15}$ and a variation of gravitational redshift of $4.8 \times 10^{-10}$, one could get a relative error of $3.8 \times 10^{-6}$ on the violation of LPI. We will see in the next section that we can perform such a test with the eccentric Galileo satellites. However the errors coming from position and velocity uncertainties will be higher because there is no two-way transfer, so they impact directly the determination of the onboard clock frequency.

\subsection{Galileo satellites Doresa and Milena}

Galileo satellites Doresa and Milena (or 5\&6) satellites were launched on August, 30th 2014. Because of a technical problem, the launcher brought them on a wrong, elliptic orbit. An elliptic orbit induces a periodic modulation of the gravitational redshift (Ashby 2003). With the good stability of recent GNSS clocks one can then test this periodic modulation to a very good level of accuracy. The Galileo 5 and 6 satellites, with their large eccentricity and on-board H-maser clocks, are hence perfect candidates to perform this test. Contrary to the GP-A experiment, it is possible to integrate the signal on a long duration, therefore improving the statistics. Data collection is ongoing and we expect first result of this experiment in 2016/2017. In the following, we assess 
the sensitivity of the gravitational redshift test that can be performed with Galileo satellites 5 and 6 using numerical simulations. This is based on article (Delva 2015).

\section{a) Description of the experiment}

The experiment proposed in (Delva 2015) requires an accurate knowledge of the frequency of the satellite clock as it orbits the Earth. These data are made available by several analysis centers (ACs) of the International GNSS Service, IGS (Dow 2009) in the framework of the Multi-GNSSExperiment, MGEX (Montenbruck 2013). MGEX applies the standard IGS processing chain to new GNSS (Galileo, Beidu) and regional services (e.g., QZSS for Japan). All active Galileo satellite orbits, clock corrections, and inter-system biases are then available for public use through the MGEX product directory at the IGS data center CDDIS, which is updated on a regular basis.

Since each $A C$ is following a different strategy, as an example we shall focus on the processing performed at the CODE (Center for Orbit Determination in Europe) analysis center (Dach 2012). In this particular case, the whole solution is based on a two steps process. First, satellite orbits are determined using a double difference network solution. This technique allows to eliminate or reduce several biases by combining observations among a pair of receivers and a pair of satellites. All clock errors are eliminated in the combination while the remaining parameters (including ephemerides, atmospheric and antenna parameters, etc...) are estimated for all stations and satellites involved. The latter are then used as a framework for the clock solution, which is based on a zero-difference processing. For more general information about the IGS and its products and data centers, the reader can refer to (Dow 2009).

For the test proposed in (Delva 2015), the orbit solution of Galileo 5 and 6 satellites would be used to calculate the behavior of the on-board clocks and the gravitational redshift as predicted by GR (Will 1993, 2014). The latter would then be compared to the clock solution from the IGS processing to recover any violation of the LPI. Several conditions need to be satisfied by the data to assure the success of the experiment. The availability of continuous orbit and clock parameters is paramount and is assured by the IGS guidelines for the clock and orbit products. Orbits and station coordinates should be unaffected by a putative violation of the gravitational redshift. This is assured as a result of the processing outlined above, since both are derived by a doubledifference solution which eliminates all clock parameters. Finally, the reference clock for the solution is always chosen among ground station H-maser clocks (Bock 2009), so that it is unaffected by a possible LPI violation. Below we study the attainable sensitivity of the test using simulated data.

\section{b) Simulation: orbits, clocks and signal}

Orbital parameters of Galileo satellites 5 and 6 used for the simulation of the present work are summarized in table 2 . Their orbits have been circularized, from initial eccentricity e $=0.2330$ to $\mathrm{e}=0.1561$. However, we will consider both the initial and the final orbits, in order to compare the sensitivity of the redshift test at different eccentricities. Orbits are calculated by solving the Kepler equation for a duration of two years, thus ensuring the numerical stability of the solution with time. 
We use the clock solutions provided by CODE to simulate a realistic random noise for Galileo satellite clocks. Fig. 8 shows the Modified Allan Deviations (MDEVs) for several GPS and Galileo

\begin{tabular}{c|c|c|c} 
Name & $e$ & $a(\mathrm{~km})$ & $i\left(^{\circ}\right)$ \\
\hline Galileo 5\&6 Final Orbit & 0.1561 & 27977 & 49.7212 \\
Galileo 5\&6 Initial Orbit & 0.2330 & 26192 & 49.7740
\end{tabular}

Table 2: Orbital parameters of satellites Galileo 5 and 6 (ESA, personal communication). Their orbits were circularized so that they can be used for positioning. Both orbits are now very

clocks (Prange 2014) around Day Of Year (DOY) 100/2013, as well as the MDEV of the simulated clock noise (in blue). For an integration time of $1000 \mathrm{~s}$, the MDEVs are between $3 \times 10^{-14}$ and $7 \times 10^{-14}$, and the MDEV of the simulated clock noise is around $5 \times 10^{-14}$. The MDEVs decrease like $\tau^{-1 / 2}$, which is characteristic of white frequency noise. There is a bump at about $21000 \mathrm{~s}$ due to systematic effects. Moreover, we add a flicker noise to the simulated clock noise at $8 \times 10^{-15}$ in the MDEV (flat part of the blue curve on Fig. 8. Although Fig. 8 shows no sign of an increase of noise at large averaging times (indicative of random walk frequency noise or frequency drift), on ground measurements of Galileo H-maser clocks have shown such behavior (Rochat 2012). The latter is anyway not exceeding $5 \times 10^{-15}$ in MDEV for measurements up to $10^{5} \mathrm{~s}$ averaging time. At the frequency of our expected periodic signal $\left(2.1 \times 10^{-5} \mathrm{~Hz}\right)$, the corresponding noise is about an order of magnitude below our noise model, and we will therefore neglect it in our analysis.

The ideal signal $y$ is the gravitational part of the relative frequency difference between a ground clock $\mathrm{g}$ and the satellite clock $\mathrm{s}$ :

$$
y=\frac{G M}{c^{2}}\left(\frac{1}{r_{g}}-\frac{1}{r_{s}}\right)
$$

$r_{\mathrm{g}}$ and $r_{\mathrm{s}}$ are respectively the norm of the position vectors of the ground clock and of the on- 


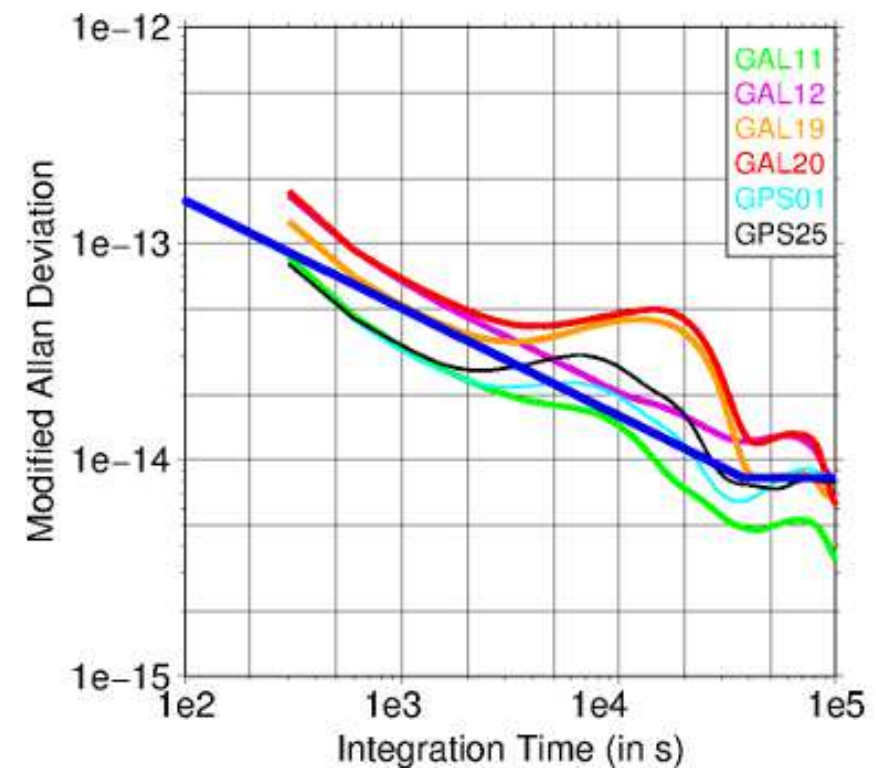

Figure 8: MDEV for Galileo In-Orbit Validation (IOV) (GAL11-12-19-20) and for GPS Block II-F (PRN GPS01-25) from (Prange 2013). Superimposed in blue is the MDEV of the simulated clock noise.

board clock. We neglect the noise coming from the ground clock, and we are interested only in the variable part of $y$. Therefore one can remove the first term in (19), which is constant. We use a simple phenomenological model for a possible violation of the gravitational redshift, characterized by a parameter $\alpha$ which is zero in general relativity (Will 1993, 2014):

$$
\tilde{y}(\alpha)=-(1+\alpha) \frac{G M}{c^{2} r_{s}} .
$$

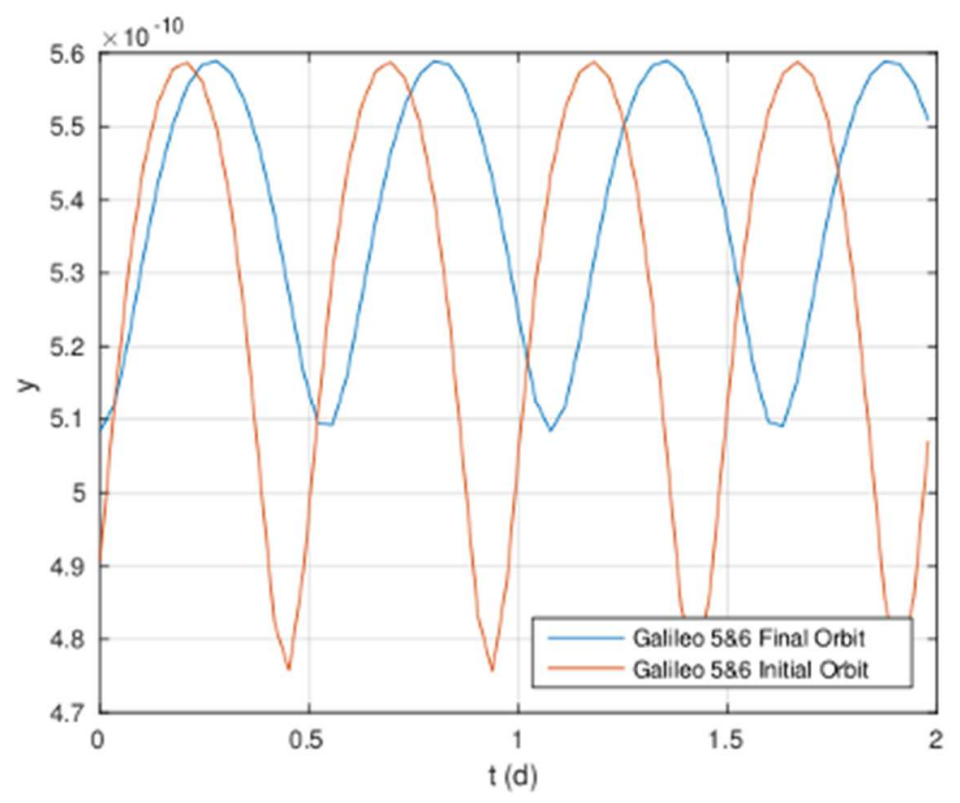

Figure 9: Gravitational redshift signal with $\alpha=0$. 
The GP-A limit corresponds to $\sigma_{\alpha}=1.4 \times 10^{-4}$ (Vessot 1989), where $\sigma_{\alpha}$ is the uncertainty on the estimation of the parameter $\alpha$.

The simulated signal $y$ is shown in Fig. 9. Its amplitude is around twice as large for Galileo satellites $5 \& 6$ initial orbits than for Galileo 5\&6 final orbits. The asymmetry between the upper part and the bottom part of the curves is characteristic of the elliptical orbit.

\section{c) Estimation of the statistical sensitivity of the test}

We study the sensitivity of the gravitational redshift test performed with Galileo 5 and 6 through two very different methods: the Fast Fourier Transform (FFT) method and the Linear LeastSquare (LSQ) method.

In the FFT method the sensitivity of the gravitational redshift test is calculated as the inverse of the Signal-to-Noise Ratio (SNR) $\rho$. The SNR can be maximized with matched filtering, i.e. by using a filter optimized for the searched signal and the considered noise. Then the SNR is given by (Gardner 1990):

$$
\rho^{2}=\int_{-\infty}^{+\infty} \frac{|F[y](f)|^{2}}{s_{\epsilon}(f)} d f
$$

where $y$ and $\epsilon$ are the gravitational redshift signal and the clock noise, respectively, $F[y]$ is the Fourier transform of $y$, and $S_{\epsilon}$ is the Power Spectral Density (PSD) of the clock noise. We implemented a numerical version of this method with the following steps: first we generate 100 different sequences of clock noise; we calculate the PSD of each sequence using a fast Fourier transform algorithm and average the 100 different PSD; finally we compute the Signal-to-Noise Ratio (SNR) (21) with the mean PSD of the clock noise, by integrating in the frequency domain $\left[-F_{s} / 2, F_{s} / 2\right]$, where $F_{s}$ is the sampling rate of the signal. This is done for several different durations of the experiment.

The second method implemented consists in using a linear least-square (LSQ) fit combined with a Monte-Carlo method, where we find the minimum of the merit function

$$
\chi^{2}=\sum_{i=1}^{N}\left[\left(y\left(t_{i}\right)+\epsilon_{i}\right)-\left(\tilde{y}\left(\alpha ; t_{i}\right)+A\right)\right]^{2},
$$

with respect to $\alpha$ and $A$. In Eq. (22) $N$ is the number of simulated observables, $A$ is a constant to be estimated and $\left(\epsilon_{i}\right)_{i=1 . . N}$ is one sequence of simulated clock noise, while $\left(y\left(t_{i}\right)+\epsilon_{i}\right)$ corresponds to the simulated observable at time $t_{i}$. The constant $A$ is introduced to remove a constant frequency bias which cannot be measured accurately. Instead of fitting $A$, it is also possible to remove the mean of $\tilde{y}(\alpha)$ from the model, so that the fitted model has a zero mean, like the noise $\epsilon$. We implemented a numerical version of this method, with the following steps: we generate 100 different sequences of clock noise (we take the same ones as for the FFT method); we estimate $\alpha$ for each clock noise sequence with the linear LSQ method, for different durations of the experiment; finally, for each duration, we calculate the mean of the 100 obtained 
values of $\alpha$ and their standard deviation $\sigma_{\alpha}$. The standard deviation corresponds approximately to $68 \%$ of the obtained values around the mean.

Following (Arun 2005) it can be shown that for high SNR and in the case of a one parameter determination, the inverse of the SNR found with matched filtering method is equal to the standard deviation of $\alpha$ found with the LSQ method, i.e. $\sigma_{\alpha}=\rho^{-1}$. Fig. 10 shows the statistical sensitivity of the gravitational redshift test, $\sigma_{\alpha}$, with respect to the duration of the experiment for the two different methods presented above and for the final and initial orbits of Galileo

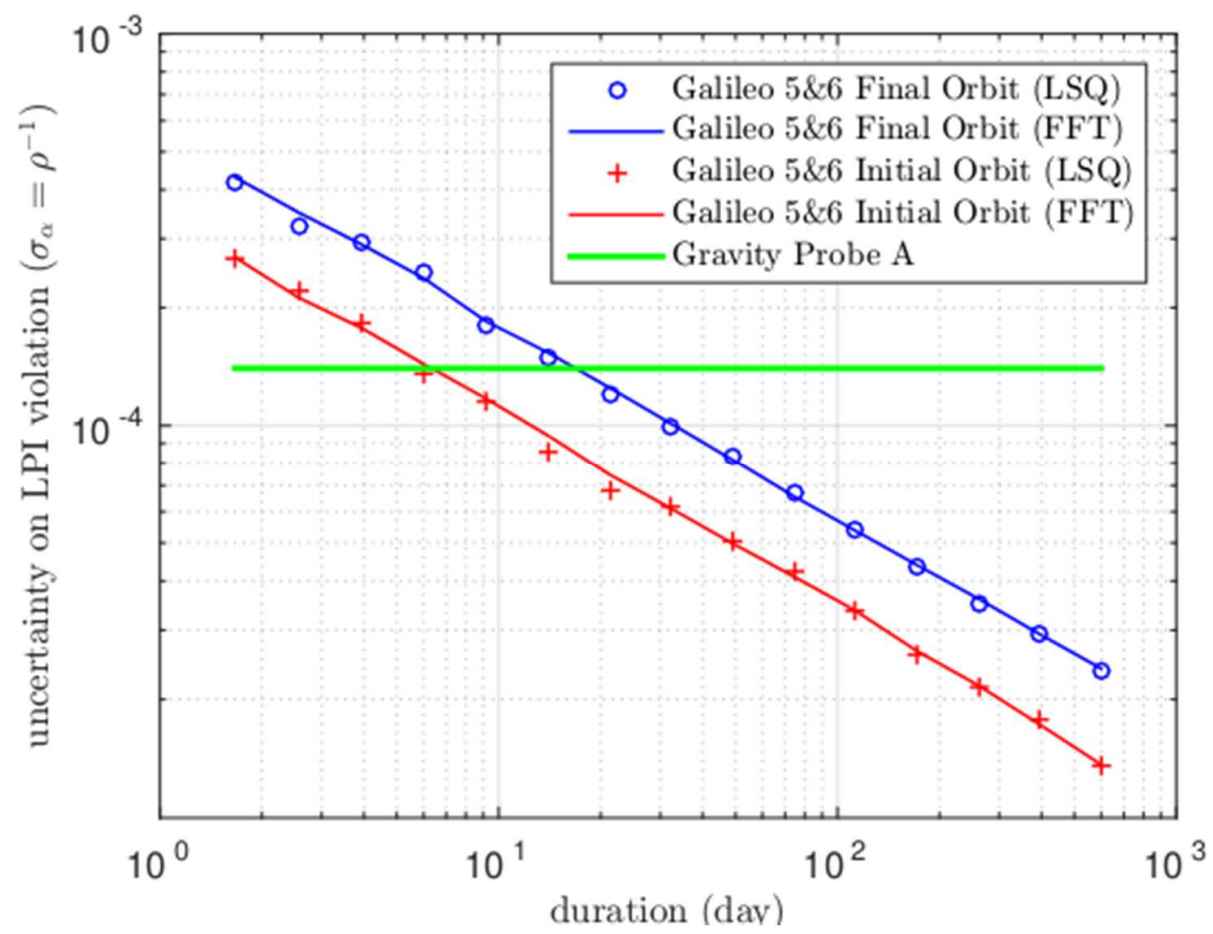

Figure 10: Statistical sensitivity of the gravitational redshift test with respect to the duration of the experiment. We show the results for the two methods FFT and $L S Q$, and for the initial and final orbits of Galileo satellites 5\&6. In green is the limit from the Gravity Probe A experiment for reference.

satellites $5 \& 6$. Also, we verify that the two methods give the same estimate, thus giving us strong confidence in the reliability of our analysis. This result is very promising, as it can be seen that even with the less eccentric orbit, the GP-A limit could be attained in less than a month. However, this result has to be tempered with a study of systematic effects.

\section{d) Systematic effects}

All systematic effects (i.e. errors that are due to unmodeled deterministic effects) that mimic the gravitational redshift signal may induce a bias in the estimation of $\alpha$ i.e. a "fake" violation of the gravitational redshift. This will ultimately limit the useful duration of noise averaging presented in Fig. 10. The bias on the estimated value of $\alpha$ resulting from unmodeled systematic effects will depend on their magnitude, frequency and phase. We classify systematic effects in four classes: (i) effects acting on the frequency of the reference ground clock; (ii) effects on the links, e.g., mismodeling of ionospheric or tropospheric delays, variations of receiver/antenna delays, 
multipath effects, etc... ; (iii) effects acting directly on the frequency of the space clock, e.g., temperature and/or magnetic field variations on board the Galileo satellites ; (iv) orbit modelling errors. The latter directly translate into variations of the measured clock frequencies because the clock solutions are obtained from the one-way signals (so called "zero differences"), hence an orbit error of $\Delta x$ along the line of sight leads directly to an error in the clock phase estimation of $\simeq \Delta x / c$.

Effects on the ground reference clock are likely to be negligible with respect to the others, and are furthermore likely to be only weakly correlated with the signal we are searching for shown in Fig. 9. They will therefore be neglected in this analysis.

Effects on the link could be significant for certain stations (e.g. under unfavorable multipath environment or atmospheric conditions). However they are expected to vary with elevation and azimuth, and local meteorological conditions, none of which are correlated with the expected signal. Note also that the main signal frequency is $2.1 \times 10^{-5} \mathrm{~Hz}$ whereas the 2 nd harmonic of any diurnal effect (temperature, humidity etc...) is $2.3 \times 10^{-5} \mathrm{~Hz}$, which indicates that such effects will decorrelate from the signal with as little as 6 days of data. Finally, some significant averaging can be expected given the large number of observing stations.

The environmental sensitivity of the Galileo passive hydrogen masers (PHM) has been characterized on ground (see e.g. Rochat 2012). Temperature sensitivity is $\lesssim 2 \times 10^{-14} / \mathrm{K}$ and magnetic field sensitivity $\lesssim 3 \times 10^{-13} / \mathrm{G}$. Both Galileo 5 and 6 are equipped with on-board temperature sensors, but no in-situ temperature measurements are publicly available. From Fig. 9 we see that the expected peak to peak variation of the redshift signal is about $5 \times 10^{-11}$. Given the expected statistical sensitivity of our test (cf. Fig. 10) we want any systematic effect to remain $2 \times 10^{-5}$ below that level, i.e. $\lesssim 10^{-15}$. This implies that temperature variations at the clock that are correlated with the signal need to be $\lesssim 0.05 \mathrm{~K}$ peak to peak. Whether this is achieved in orbit remains to be seen once temperature measurements are made available. As an example, Montenbruck (2012) mention that for the GPS block IIF "the rubidium clocks of the Block IIF satellites are mounted on a thermally isolated sub-panel (along with the frequency distribution unit) for which a peak-to-peak temperature variation of less than $0.5 \mathrm{~K}$ has been observed in SVN62 on-board measurements. In addition, the new clock benefits from a baseplate temperature controller (BTC), which further reduces the sensitivity to external temperature variations by up to a factor of 50." This indicates that peak to peak variations $\lesssim 0.05 \mathrm{~K}$ are realistic. Additionally, significant decorrelation between temperature effects and our signal can be expected, as the former depend on Sun exposure of the satellite and thus have annual spectral components, which are absent in the signal. To evaluate the influence of the magnetic effect on our determination of $\alpha$ we model the Earth magnetic field as a dipole with the field amplitude at the location of the satellite given by

$$
|B|=B_{0}\left(\frac{R_{E}}{r}\right)^{3} \sqrt{1+3 \cos ^{2} \vartheta}
$$


where $B_{0}=0.312 \mathrm{G}, R_{\mathrm{E}}$ is the mean radius of the Earth and $\vartheta$ is the angle between the position vector of the satellite and the geomagnetic North pole. We then use the clock sensitivity coefficient to add a systematic effect $\epsilon_{s y s}(t)$ to our signal, where the time dependence is given by (23) and by the satellite orbit. We determine $\alpha$ from the simulated signal $Y$ using the LSQ method (see above), such that

$$
Y(t)=y(t)+\epsilon_{s y s}(t)+\epsilon(t)
$$

where $\epsilon$ is the random clock noise. Fig. 11 shows the obtained value of $\alpha$ (the bias on $\alpha$ induced by the systematic effect) as a function of observation time together with the obtained uncertainty from the LSQ-Monte Carlo method. We see that for observation times up to 450 days the magnetic effect remains below the statistical uncertainty. For longer integration times it will become limiting at $\simeq 2.7 \times 10^{-5}$. This is a worst case estimation as it is of course possible to correct at least partly for the magnetic effect and/or simultaneously fit the magnetic effect and $\alpha$ (c.f. the discussion of the solar radiation pressure effect below) leading to a better result.

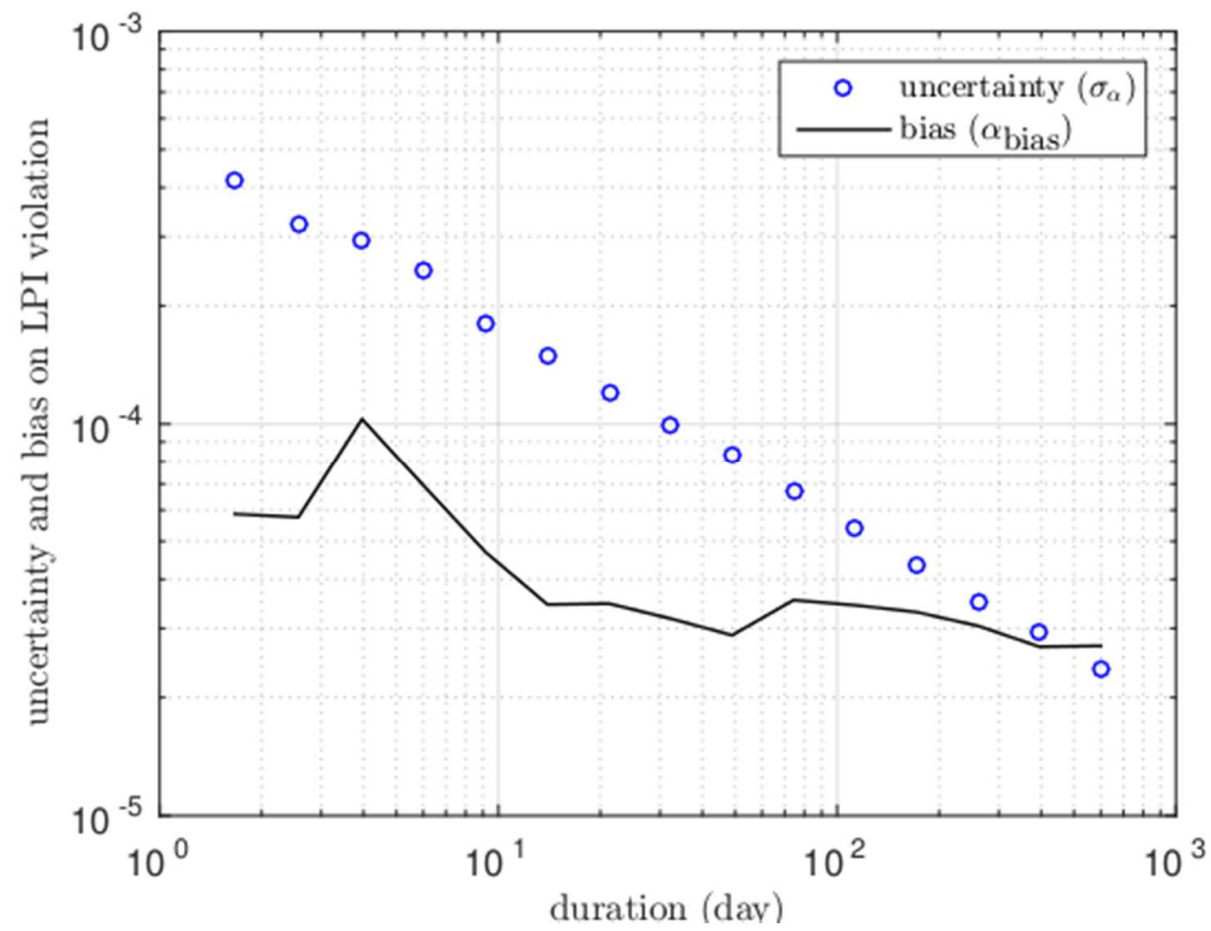

Figure 11: Statistical uncertainty (blue circles) on the LPI violation and bias induced by uncorrected magnetic effect (black line) as a function of observation time, for Galileo $5 \& 6$ final orbits.

Finally, we look at effects related to orbit modelling errors. The radial part of the resulting orbit error contributes directly to the clock estimation error. Indeed, it has been shown that the clock offset is correlated with satellite laser ranging (SLR) residuals, which can be considered as a measure of the radial orbit error (Montenbruck 2014). The clock error also shows a dependency with the Sun elevation angle, which is the angle between the satellite orbital plane and the direction of the Sun. This clearly indicates systematic effects which are linked to the direction of 
the Sun, like a mismodeling of Solar Radiation Pressure (SRP) (Montenbruck 2014). Indeed, the use of an enhanced SRP model for orbit determination can divide by a factor of four the effect of SRP mismodeling on the clock estimation. The resulting systematic effect on the clock estimation is evidenced as a bump at half-orbital time scale in the Modified Allan Deviation (MDEV) of Fig.8. Consequently, we will consider here a class of systematic effects which shows a dependency with the Sun direction, as well as with the mean anomaly of the satellite. For the SRP effect in particular we use a model that reproduces the annual amplitude variation (cf. Fig. 3 of Montenbruck 2014) and has a frequency of $n+\omega_{a}$, where $n$ is the mean motion of the satellite and $\omega_{a}=2 \pi /$ year :

$$
\epsilon_{s y s}(t)=A\left(1+B\left(\cos \left(\omega_{a} t+\phi_{1}\right)-1\right)\right) \sin \left(\left(n+\omega_{a}\right) t+\phi_{2}\right)
$$

with $A, B, \phi_{1}, \phi_{2}$ constant. We simulate data with $A=5 \times 10^{-14}, B=0.45, \phi_{1}=0$ that reproduce the results of Montenbruck (2014), and different values of $\phi_{2}$. We then use a Bayesian approach ${ }^{1}$ with a slice sampling method (Neal 2003) assuming normal distributions and flat priors to simultaneously determine the four parameters of the SRP model (25) and the redshift parameter

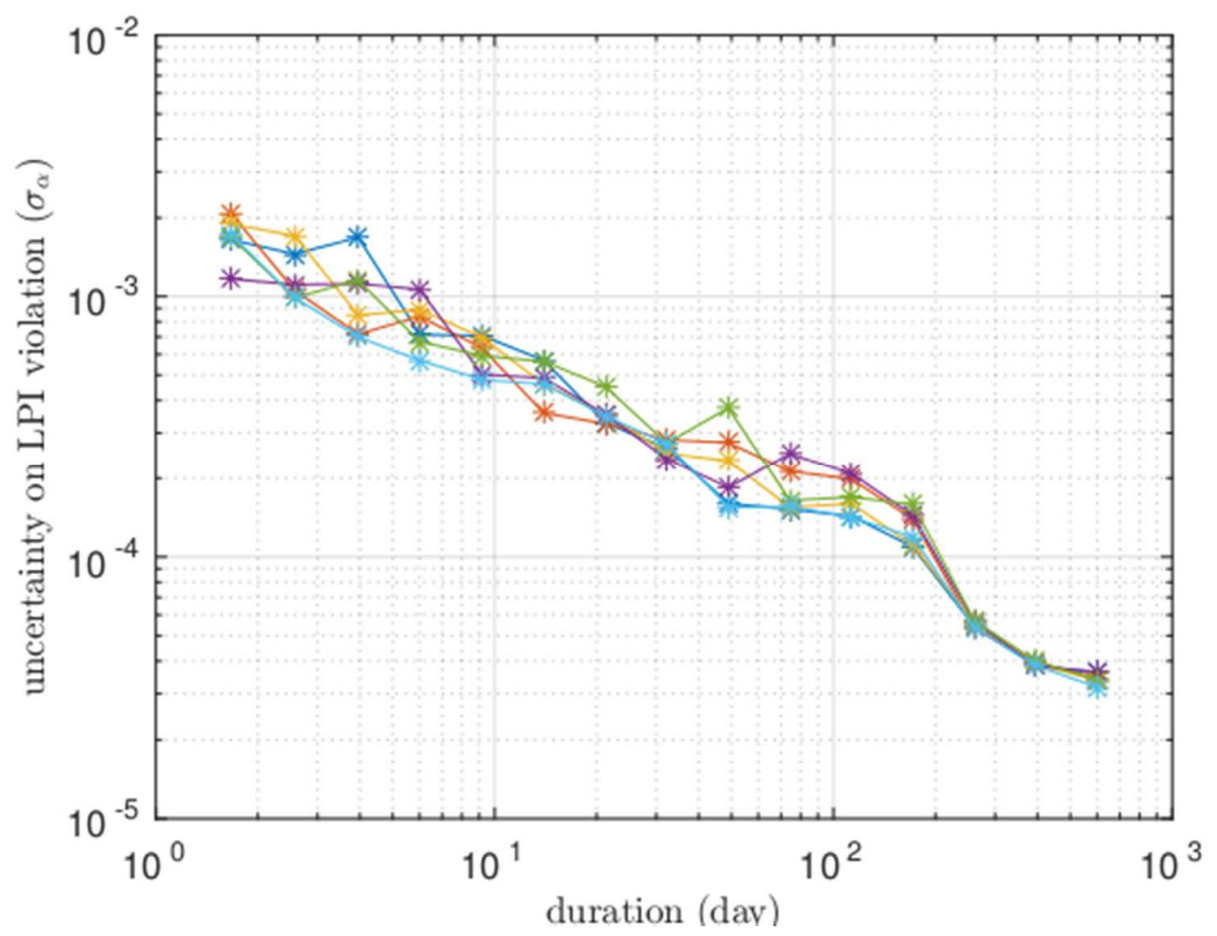

Figure 12: Statistical uncertainty of the determination of the gravitational redshift in the presence of solar radiation pressure, as a function of observation time, for Galileo $5 \& 6$ final orbits, and for six different occurrences of the simulated clock noise.

$\alpha$, as well as their respective uncertainties. The result is shown in Fig. 12 for six different occurrences of the simulated clock noise. We find that for data spanning more than 1 year, and a "worst case" choice of $\phi_{2}$ the resulting value of $\alpha$ is unbiased with a statistical uncertainty of 
$\simeq 4 \times 10^{-5}$, slightly increased with respect to the uncertainty when determining $\alpha$ with no SRP effect present in the observable (c.f. Fig. 10). After 200 days of integration time, the six occurrences of noise converge to the same values which indicates that the uncertainty is limited by the residual SRP systematic effect. In our estimates we have assumed the "standard" SRP corrections rather than the improved ones discussed in Montenbruck (2014). The latter should allow a reduction of the effect by a factor 2-4 (Montenbruck 2014), so our estimates are likely to be again only an upper limit.

\subsection{ACES / PHARAO}

ACES/PHARAO (Atomic Clock Ensemble in Space / Projet d'Horloge Atomique par Refroidissement d'Atomes en Orbite) is an international space mission of the French and European space agencies (ESA and CNES). It is an international scientific and industrial collaboration with French lead (LKB and SYRTE) that aims at realizing a time scale of high stability and accuracy on board the International Space Station (ISS). The ACES/PHARAO payload will be launched with Space-X and installed onboard the ISS in 2018, with a planned mission duration of 18 months to 3 years.

The on board fractional frequency stability (ADEV) should be better than $\sigma_{y}=10^{-13} \times \tau^{-1 / 2}$, which corresponds to $3 \times 10^{-16}$ after one day of integration. Time deviation (TDEV) should be better than $\sigma_{\mathrm{x}}=4.1 \times 10^{-14} \times \tau^{1 / 2} \mathrm{~s}$, which corresponds to $12 \mathrm{ps}$ after one day of integration (see figure 13). Absolute frequency accuracy should be around $10^{-16}$ in fractional frequency. To achieve these performances, its payload includes the first cold atom clock in space, PHARAO, which is a Cs clock, and a H-maser. Another key element is the microwave link (MWL) that uses
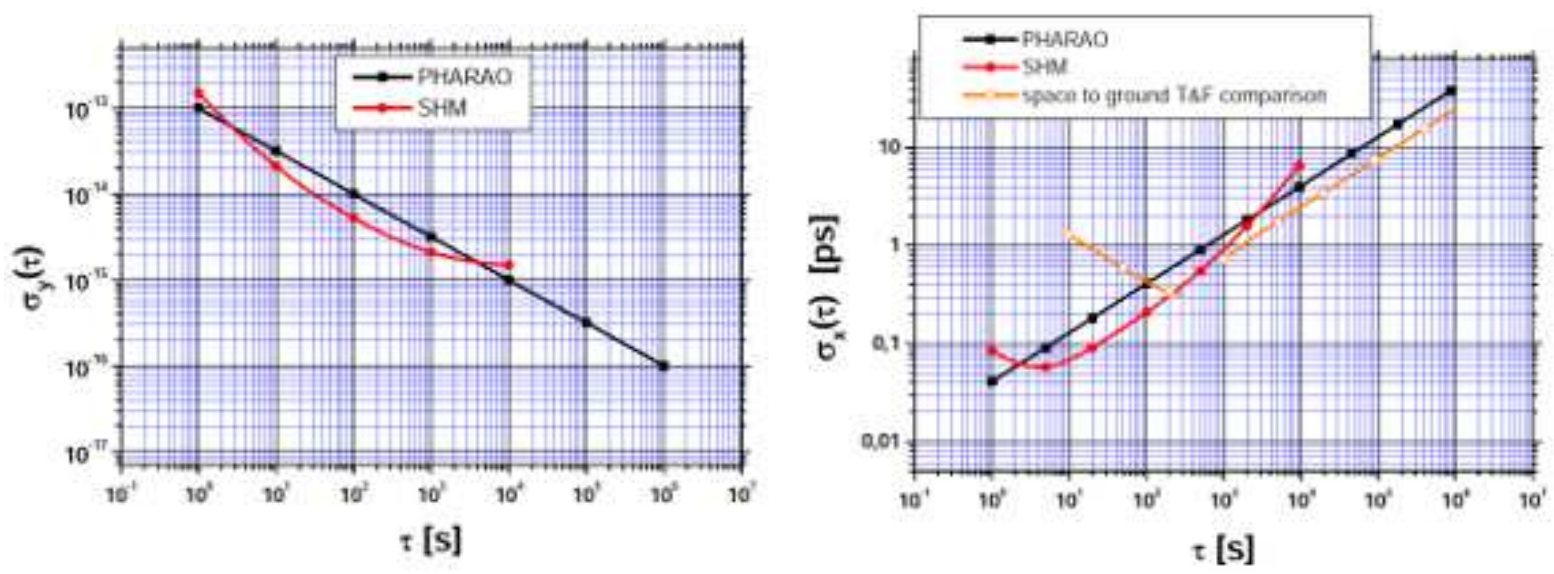

Figure 13: Left: fractional frequency stability (ADEV) of PHARAO and Space Hydrogen Maser (SHM). Right: Time stability (TDEV) of PHARAO, SHM and the space-ground time/frequency comparison link.

radio ( $\mathrm{Ku}$ and $\mathrm{S}$ band) in a two-way configuration to compare space to ground clocks at unprecedented stability and accuracy. 
The scientific objectives are:

- Demonstrate the high performance of the atomic clocks ensemble in the space environment and the ability to achieve high stability space-ground time and frequency transfer.

- Perform space to ground clock comparisons with high resolution on a world-wide basis using a link in the microwave domain. The link stability should reach around $0.3 \mathrm{ps}$ after $300 \mathrm{~s}$ of integration and around 7 ps after 1 day of integration.

- Perform tests of the Einstein Equivalence Principle (EEP). It will be possible to test Local Lorentz Invariance (LLI) and Local Position Invariance (LPI) to unprecedented accuracy by doing three types of tests: a test of the gravitational redshift, contribute to the search for a variation of fundamental constants, an LLI tests in different theoretical framework (e.g. the SME, see section 2).

Besides these primary objectives, several secondary objectives can be found in (Cacciapuoti 2009). For example, the measurement of gravitational redshift can be used to measure gravitational potential differences between different clock locations, which is a new type of geodetic measurements using clocks, called chronometric geodesy (Delva 2014).

LKB and SYRTE are heavily involved in the experiment, from the conception of the cold atom clock to the development and the data analysis. SYRTE is an official ACES Data Analysis Center. Presently we develop a data analysis algorithm in order to process the mission raw data and extract the scientific products of the mission, which are mainly the desynchronization between clocks. In order to test our algorithm, we developed a full simulation of the raw observables, as they will be produced by the MWL modem and instrumentation.

A violation of LPI can be quantified as described in the previous section by a factor $\alpha$ appearing in the standard formula of gravitational redshift. The most stringent limit from previous measurements comes from the 1976 NASA/SAO GP-A rocket experiment (Vessot 1989), yielding $|\alpha| \lesssim 1.4 \times 10^{-4}$. This test took advantage of the stability of the spaceborne and ground hydrogen maser clocks, connected by a continuous-wave microwave link, to measure the modulation of the gravitational redshift with altitude. On the contrary, the gravitational redshift in the ACES experiment will only be modulated to around 10\%; therefore the high accuracy of the spaceborne $\left(10^{-16}\right)$ and ground clocks are used, to measure the absolute redshift which will constrain $|\alpha| \lesssim 3 \times 10^{-6}$, an improvement by a factor 45 compared to the GP-A experiment.

High accuracy comparisons of atomic frequency standards based on different atoms and ions can be interpreted as a test of the variation of fundamental constants. By combining the comparisons between different types of atomic clocks (eg. $\mathrm{Rb} / \mathrm{Cs}, \mathrm{Al}+/ \mathrm{Hg}+, \mathrm{Hg}+/ \mathrm{Cs}, \ldots$ ), it is possible to disentangle the contributions coming from three different fundamental constants: $\alpha_{e m}$ the fine structure constant (electroweak interactions), $\mu=m_{e} / m_{p}$ the electron-to-proton mass ratio, and $\mathrm{m}_{\mathrm{q}} / \Lambda_{\mathrm{QCD}}$ the quark mass scaled to the quantum chromodynamics mass scale $\Lambda_{Q C D}$ (strong interactions). Present limits can be found in (Guéna 2012). The ACES experiment will improve these limits by increasing the number of possible inter-clock comparisons from distant laboratories. 
Finally, the ACES experiment will test LLI by testing the independence of the clock synchronization procedure with the orientation of the microwave link. In this experiment, one compares the rest transition frequency of an atom, to its transition frequency when at high velocity as seen by an observer at rest. The expected sensitivity is expected to be comparable to the best present limit obtained from an Ives-Stilwell type experiment (Botermann 2014). Both types of tests are complementary.

A test of LLI in the SME can also be done with PHARAO following the test realized on Earth in (Wolf 2006). Beyond a faster integration due to the shorter orbital period as already mentioned, the time dependence of the signal will allow a more constraining set of measurements for SME coefficients: the quantization axis of PHARAO being along the ISS trajectory, over one orbital period its direction will be fully reversed twice, giving rise to a comparatively rapid modulation of the expected signal.

\subsection{The Cassini test of post Newtonian gravity}

On its cruise phase to Saturn the Cassini mission was used to carry out what is still today the most precise test of post-Newtonian gravity in the solar system (Bertotti 2003). It is a test of the Einstein field equations in the parametrized post-Newtonian (PPN) framework that was described in section 2.2. In that framework it sets the most stringent limit on the $\gamma$ parameter at $\gamma-1=(2.1 \pm 2.3) \times 10^{-5}$. Additionally to providing the best constraint in the solar system on that parameter, the Cassini experiment has also been used at galactic and cosmological scales to provide combined constraints on theories at large scales (c.f. section 2.3). The experiment measured the travel time of radio signals between the NASA deep space network (DSN) antennas and the Cassini spacecraft as the line of sight approached the $\operatorname{limb}$ of the sun. The good performance of the atomic clocks in the NASA deep space network (DSN) were key to its success.

\section{a) Principle of the experiment}

During its cruise phase to Saturn the Cassini spacecraft passed almost behind the sun (as seen from the Earth) on $21^{\text {st }}$ June 2002. During the hours before and after closest approach the Radio links between the Earth antennas of the NASA deep space network (DSN) and the Cassini spacecraft were used to precisely measure the frequency shift of radio signals sent to the spacecraft and back. In general relativity as well as all metric theories of gravitation, e.g. PPN, and electromagnetic signal experiences a gravitational delay (also known as Shapiro delay) when passing close to a gravitating body. That delay can be measured by monitoring the return travel time of the signal as the line of sight to the reflector passes close to a gravitational source. The derivative of that delay induces a frequency shift $y=\frac{\Delta f}{f}$ in the signal given by

$$
y(t)=-4(1+\gamma) \frac{G M_{S}}{c^{3}} \frac{1}{b(t)} \frac{d b(t)}{d t}
$$


where, $M_{s}$ is the mass of the sun, $b(t)$ is the impact parameter (point of closest approach) of the light signal and $\gamma=1$ in GR. The factor 2 difference with respect to equ. (2) of Bertotti (2003) is a misprint in (Bertotti 2003), which however has no further consequences in the analysis there. The point of closest approach occurred with a minimal impact parameter of 1.6 solar radii.

\section{a) Results}

The DSN data was fitted with a 12 parameter model that includes $\gamma$, the six initial conditions of the spacecraft trajectory, three parameters for the non-gravitational acceleration due to thermal emissions, and two parameters characterizing the reflectivity of the spacecraft antenna (to correct for solar radiation pressure). The main noise sources were measurement noise including that of the DSN clocks. Systematic effects from dispersion (solar corona and Earth's ionosphere) and troposphere were removed from the data using a dispersion free (to first order) combination of $\mathrm{Ka}$ and $\mathrm{X}$ band signals, and modeling of the troposphere from meteorological data taken at the DSN sites. No estimate on the residuals dispersion and tropospheric effects, that could mask a putative violation of GR, are available in (Bertotti 2003). The other main source of systematic errors is the non-gravitational acceleration of the spacecraft due to thermal radiation and solar pressure. This was accounted for by fitting appropriate parameters in the global model. Additionally, in order to exclude possible effects from mismodelling, different models were used yielding all similar results. The final result of Bertotti (2003) is then

$$
\gamma-1=(2.1 \pm 2.3) \times 10^{-5}
$$

With the uncertainty entirely due to statistics and limited by measurement noise, which includes the noise of clocks in the DSN stations.

\section{Conclusion and Outlook}

We conclude this chapter by describing briefly some space projects in the more distant future that will probe fundamental physics using high accuracy clocks on ground and in space.

The Space Optical Clock (SOC) proposal aims at putting an optical lattice clock onboard the International Space Station (ISS) in a 2020-2025 time frame (Bongs 2015, Origlia 2016). Additionally the mission is planned to carry an improved version of the Microwave link (MWL) of ACES as well as an optical link for high performance space-ground clock comparisons. The objectives are similar to the ACES mission objectives (see above) but with an improvement in performance by about a factor 10 . That improvement is expected to come from the accuracy of the clock (at the $10^{-17}$ level in fractional frequency) and the better performance of the time/frequency transfer links. Currently the project is undergoing a definition and assessment (phase A) study at ESA, expected to last until late 2017.

The gravitational time delay experiment (Ashby 2010) proposes to measure the gravitational time delay of an electromagnetic signal to unprecedented accuracy. When interpreted in the PPN 
framework (see section 2.2) it will set a limit on the PPN parameter $\gamma$ of $\gamma-1 \leq 10^{-8}$. This corresponds to an improvement on the state of the art (see above) by over three orders of magnitude. The principle of the mission is shown on figure 14. A laser link between two spacecraft on either side of the sun is used to measure the return travel time of light signals. One of the spacecraft carries a highly stable optical clock, the other one a laser transponder that returns the signal. Both spacecraft are drag-free controlled thereby avoiding effects coming from non-gravitational accelerations.

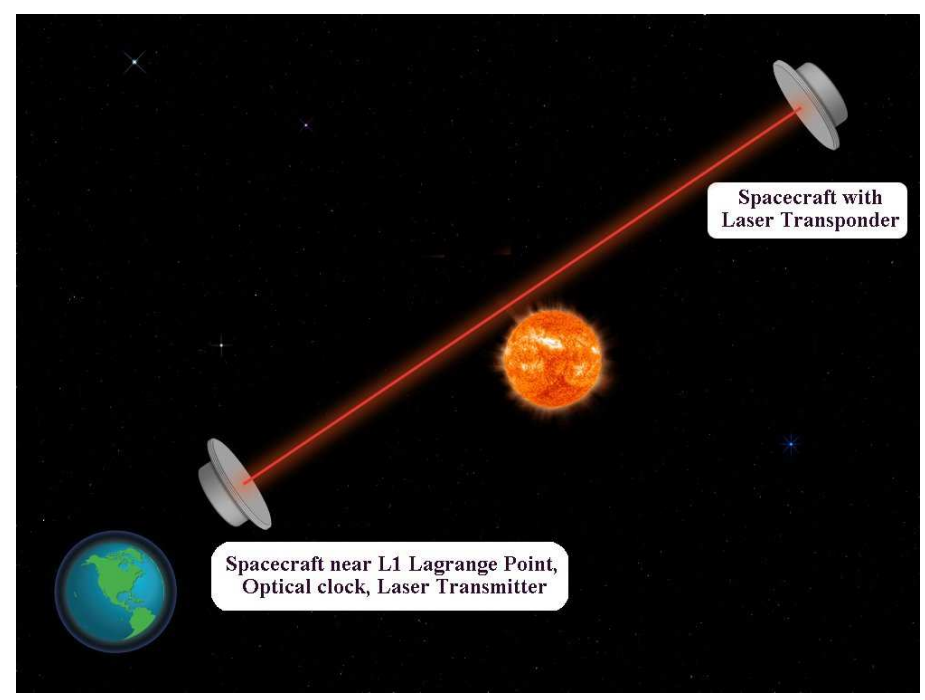

Figure 14: Principle of the gravitational time delay experiment

Extensive simulations were carried out to study the performance of such a mission. The baseline of those simulations was a 5 year mission, with three occultation events 1,3 and 5 years after launch. The assumed clock stability of $1 \times 10^{-15} / \sqrt{ } \tau$ (Allan deviation), which is already surpassed by best ground clocks. The drag free performance assumed in the simulations was $10^{-13} \mathrm{~m} / \mathrm{s} 2 / \sqrt{\mathrm{Hz}}$, which is an order of magnitude less demanding than the recently demonstrated performance of the LISA-Pathfinder spacecraft (Armano 2016). Finally the measurement noise of the laser returntime measurement was assumed at $0.02 \mathrm{ps} / \sqrt{\mathrm{Hz}}$, which is more than six orders of magnitude less demanding than e.g. the laser link of the LISA mission.

Although similar in principle to the Cassini experiment described in the previous section, a number of modifications will allow significant improvements. Apart from the lower noise levels of the measurement systems, the absence of the atmosphere, drag-free spacecraft and optical frequency of the signal (rather than radio) should allow better control of the main systematics. In particular the optical frequency will strongly decreases the effect of the solar corona and will therefore allow a minimization of the impact parameter, which increases the signal.

In summary, the mission is expected to allow a test of the Einstein field equations at unprecedented levels. In the PPN framework it will lead to an improvement on the knowledge of the $\gamma$ parameter by over three orders of magnitude. It requires only state of the art performance 
of present day technology. The main challenge will be the making that technology, and in particular the optical clock, sufficiently small and robust for space operation.

E-GRIP (Einstein Gravitational Redshlft Probe) is a study case of a fast, relatively cheap and high TRL mission to test the Einstein Equivalence Principle (EEP) via the gravitational redshift of a space clock in a highly eccentric Earth orbit. The principle is very similar to the test using the eccentric Galileo 5 \& 6 satellites (see section 3.2). It aims at using well developed technology: the space hydrogen maser (SHM), Microwave link (MWL), and laser timing system (ELT) of the ACES mission (see section 3.3), adapted for the different orbit where necessary. The main gain comes from the highly elliptic orbit. Two scenarios were considered, with semi-major axes equal to $24450 \mathrm{~km}$ and $35000 \mathrm{~km}$, and eccentricities of 0.636 and 0.800 . The goal is a test of the gravitational redshift with a relative uncertainty of $4 \times 10^{-7}$, which represents an improvement by about a factor 350 with respect to the best present knowledge from the Gravity Probe-A experiment (see section 3.1), and by about a factor 5 with respect to the expected result from ACES (section 3.3). Although the latter is only a modest gain, it can be obtained from a high TRL, cheap and rapid mission.

The Space-Time Explorer and QUantum Equivalence Space Test (STE-QUEST) space mission (Altschul 2015, STE-QUEST 2013) is an M class mission proposal that was pre-selected by the European Space Agency in 2010 together with four other missions for the cosmic vision M3 launch opportunity. It carries out tests of different aspects of the Einstein Equivalence Principle using atomic clocks, matter wave interferometry and long distance time/frequency links, providing fascinating science at the interface between quantum mechanics and gravitation that cannot be achieved, at that level of precision, in ground experiments. Since 2010 STE-QUEST has been studied in different configurations and mission scenarios. The most extensive version includes two main payload elements: A two species $\left({ }^{87} \mathrm{Rb}-{ }^{41} \mathrm{~K}\right.$, or $\left.{ }^{87} \mathrm{Rb}-{ }^{85} \mathrm{Rb}\right)$ atom interferometer that carries out a test of the universality of free fall in the quantum regime (Bose-Einstein Condensates in large quantum superpositions) and an evolved version of the PHARAO cold-atom space clock from ACES (section 3.3) for tests of the gravitational redshift. Additionally it will carry an improved version of the ACES MWL and a new type of optical link for high performance ground-space time/frequency comparisons. The considered mission scenarios have highly elliptical orbits ( $700 \times 51000 \mathrm{~km}$ in the M3 version, $2500 \times 33600 \mathrm{~km}$ in the M4 version) which allow large gravitational field variations, long common view times for ground clocks when the satellite is at apogee, and strong gravitational field at perigee for the UFF test. STE-QUEST will test UFF at a level of $10^{-15}$, two orders of magnitude better than the best present result using macroscopic test-objects, and at least 6 orders better than present UFF tests in the quantum regime. It will also improve bets present results on redshift tests (in the field of the Earth, Sun, and Moon) by up to 4 orders of magnitude. Additionally tests of Lorentz invariance in the SME framework are planned that will improve knowledge of several SME coefficients by up to 4 orders of magnitude. Thus STE-QUEST is a mission that tests all aspects of the Einstein Equivalence Principle, searching for any deviation at sensitivities well beyond what is achieved today.

In summary, fundamental physics in space is a highly active field of research that thrives on technological developments, in particular of clocks, long distance radio and optical links, and 
inertial sensors (accelerometers, gyroscopes). It is impossible to describe all missions and projects in the field, and in this chapter we have mentioned only some of the most important ones, and in particular those that rely strongly on clocks and time/frequency transfer methods to achieve their objectives. Very recently $(2015 / 2016)$ there have been two missions launched that are critical to the field: LISA-Pathfinder that demonstrated unprecedented levels of dragfree technology and performance (Armano 2016), and MICROSCOPE that will carry out the first space-test of the Universality of Free Fall (see section 2.1) improving the best present measurements by about two orders of magnitude. This shows the vitality of the field and many more exciting results are expected in the near and more distant future, which will hopefully pave the way towards the new physics beyond general relativity and the standard model of particle physics. And it is likely that high performance clocks will continue to play a major role in that process.

\section{References}

Adelberger E., et al, 2003, Annual Review of Nuclear and Particle Science 53, 77.

Adelberger E., et al, 2009, Prog. In Particles and Nuc. Physics 62, 102.

Aghion S., et al, 2013, Journal of Instrumentation 8, 8013P.

Alimi J.-M., A. Füzfa, 2007, IJMPD 16, 2587.

Alimi J.-M., A. Füzfa, 2008, JCAP 9, 14.

Altschul B., et al., 2015, Advances in Space Research, 55, 501 - 524.

Armano M., et al., 2016, Phys. Rev. Lett., 116, 231101.

Arun K.G., et al., 2005, Phys. Rev. D, 71(8):084008.

Arvanitaki A., et al, 2015, Phys. Rev. D 91, 015015.

Arvanitaki A., et al, 2016, Phys. Rev. Letters 116, 031102.

Ashby N., 2003, Living Rev. Relativity, 6:1, 2003.

Ashby N., et al, 2007, Phys. Rev. Letters 98, 070802.

Ashby N., et al, 2007a, Phys. Rev. D 75, 022001.

Ashby, N.; et al., 2010 in Relativity in Fundamental Astronomy, Proceedings IAU Symposium No.

261, 2009, S. A. Klioner, P. K. Seidelman \& M. H. Soffel, eds., 414.

Avelino P., et al, 2001, Phys. Rev. D 64, 103505.

Avilez-Lopez A., et al, 2015, JCAP 06, 044.Bai Y., et al, 2015, JCAP 10, 029.

Bailey Q., and A. Kostelecky, 2006, Phys. Rev. D 74, 045001.

Bailey Q., et al, 2013, Phys. Rev. D 88, 102001.

Battat J., et al, 2007, Phys. Rev. Letters 99, 241103.

Bekenstein J., 1982, Phys. Rev. D 25, 1527.

Bekenstein J., J. Magueijo, 2006, Phys. Rev. D 73, 103513.

Bertotti B, et al, 2003, Nature 425, 374.

Blanchet L., and J. Novak, 2011, MNRAS 412, 2530.

Bock H., et al., 2009, J. Geod., 83:1083-1094.

Bongs K., et al., 2015, Comptes Rendus Physique 16, 553-564. arXiv: 1503.08457.

Botermann B., et al., 2014, Physical Review Letters, 113(12):120405.

Bourgoin A., et al., https://arxiv.org/abs/1607.00294 
Brans C., R. Dicke, 1961, Phys. Rev. 124, 925.

Brillet A., J. Hall, 1979, Phys. Rev. Letters 42, 549.

Bruneton J.P., and G. Esposito-Farèse, 2007, Phys. Rev. D 76, 124012.

Burrage C., et al, 2015, JCAP 3, 042.

Cacciapuoti L., C. Salomon, 2009, Eur. Phys. J. - Spec. Top. 172, 57.

Carroll S., 1998, Phys. Rev. Letters 81, 153067.

Carroll S., et al, 2009, Phys. Rev. Letters 103, 011301.

Carroll S., et al, 2010, Phys. Rev. D 81, 063507.

Clifton T., et al, 2012, Phys. Reports 513, 1.

Colladay D., A. Kostelecky, 1997, Phys. Rev. D 55, 6760.

Colladay D., A. Kostelecky, 1998, Phys. Rev. D 58,116002.

Dach R., et al., 2013, Center for orbit determination in europe (CODE). Technical report, International GNSS Service: Technical Report 2012, 2013. edited by R. Dach and Y. Jean (AIUB), IGS Central Bureau.

Damour T., and A. Polyakov, 1994, Nucl. Phys. B 423, 532.

Damour T., et al, 1990, Phys. Rev. Letters 64, 123.

Damour T., J. Donoghue, 2010, Phys. Rev. D 82, 084033.

Damour T., 2012, Class. And Quantum Grav. 29, 184001.

Delva P., et al., 2014, in: S. M. Kopeikin (Ed.), Frontiers in relativistiv celectial mechanics. Volume 2: applications and experiments. De Gruyter.

Delva P., et al., 2015, Classical and Quantum Gravity, 32(23):232003.

Derevianko A., and M. Pospelov, 2014, Nature Physics 10, 933.

Dow J.M., et al., 2009, J. Geod., 83:191-198.

Einstein A., 1911, Annalen der Physik, 340, 898.

Einstein A., 1916, Annalen der Physik 154, 769

Famaey B, S. McGaugh, 2012, Living Review in Relativity 15, 10.

Fienga A., et al, 2015, Cel. Mechanics and Gyn. Astronomy 123, 325.

Fischbach E., C. Talmadge, 1999, The search for Non-Newtonian gravity, Springer.

Frieman J., B. Gradwohl, 1991, Phys. Rev. Letters 67, 2926.

Füzfa A., J.-M. Alimi, 2007, Phys. Rev. D 75, 123007.

Gardner W.A., 1990, Introduction to Random Processes: with applications to signals and systems, volume 31. McGraw-Hill New York.

Godun R., et al, 2014, Phys. Rev. Letters 113, 210801.

Gradwohl B., J. Frieman, 1992, ApJ 398, 407.

Guéna J., et al, 2012, Phys. Rev. Letters 109, 080801.

Hamilton P., et al, 2015, Science 349, 849.

Hees A., et al, 2012, Class. And Quantum Grav. 29, 235027.

Hees A., and A. Füzfa, 2012, Phys. Rev. D 85, 103005.

Hees A., et al, 2014a, proceedings of the Journées 2013 "Systèmes de Référence Spatiotemporels", 241, arXiv: 1403.1365.

Hees A., et al, 2014b, Phys. Rev. D 89, 102002.

Hees A., et al, 2014c, Phys. Rev. D 90, 124064.

Hees A., and O. Minazzoli, 2015, arXiv:1512.05233. 
Hees A., and A. Füzfa, 2015, in "Thirteenth Marcel Grossmann Meeting: On Recent Developments in Theoretical and Experimental General Relativity, Astrophysics and Relativistic Field Theories", 1140.

Hees A., et al, 2015a, Proceedings of the Annual meeting of the SF2A, 125.

Hees A., et al, 2015b, Phys. Rev. D 92, 064049.

Hees A., et al, 2015c, Gen. Rel. and Grav. 47, 9.

Hees A., et al, 2016a, arXiv:1604.08514.

Hees A., et al, 2016b, MNRAS 455, 449.

Hohensee M., et al, 2011, Phys. Rev. Letters 106, 151102.

Hohmann M., 2015, Phys. Rev. D 92, 064019.

Huntemann N., et al, 2014, Phys. Rev. Letters 113, 210802.

Iorio L., 2012, Class. And Quantum Gravity 29, 175007.

Ives H., G. Stilwell, J. of Optical Soc. Of America, 28, 215.

Jaekel M.T., S. Reynaud, 2005, Class. And Quantum Grav. 22, 2135.

Jaekel M.T., S. Reynaud, 2006, Class. And Quantum Grav. 23, 777.

Kennedy R., E. Thorndike, 1932, Phys. Rev. 42, 400.

Kesden M. and M. Kamionkowski, 2006, Phys. Rev. Letters 97, 131303.

Kesden M. and M. Kamionkowski, 2006, Phys. Rev. D 74, 083007.

Khoury J., A. Weltman, 2004a, Phys. Rev. Letters 93, 171104.

Khoury J., A. Weltman, 2004b, Phys. Rev. D 69, 044026.

Konopliv A., et al, 2011, Icarus 211, 401.

Kostelecky A., M. Mewes, 2002, Phys. Rev. D 66, 056005.

Kostelecky A., N. Russel, 2011, Rev. of Modern Physics 83, 11.

Lambert S., and C. Le Poncin-Lafitte, 2011, A\&A, 529, A70.

Lamoreaux S., et al, 1986, Phys. Rev. Letters 57, 3125.

Leefer N., et al, 2013, Phys. Rev. Letters 111, 060801.

Le Poncin-Lafitte C., et al., 2016, arXiv:1604.01663.

Lovelock D., 1971, Journal of Mahetmatical Physics 12, 498.

Lovelock D., 1972, Journal of Mahetmatical Physics 13, 874.

Mansouri R., R. Sexl, Gen. Rel. and Grav. 8,497.

Mattingly D., 2005, Living Rev. in Relativity 8,5.

Martins C.J.A.P., A.M.M. Pinho, Phys. Rev. D 91, 103501.

McMillan P.J., 2011, MNRAS 414, 2446.

Merlet S., et al, 2010, Metrologia 47, L9.

Mignard F., and S. Klioner, 2010, Proceedings of IAU symposium 261, 306.

Milani A., et al, 2002, Phys. Rev. D 66, 082001.

Milgrom M., 1983a, ApJ 270, 365.

Milgrom M., 1983b, ApJ 270, 371.

Milgrom M., 1983c, ApJ 270, 384.

Milgrom M., 2009, MNRAS 399, 474.

Minazzoli O., and A. Hees, 2014, Phys. Rev. D 90, 023017.

Mohapi N., et al, 2016, JCAP 03, 32.

Montenbruck O., et al., 2012, GPS Solut., 16(3):303-313.

Montenbruck O., et al., 2013, GPS World, 24(7):44-49. 
Montenbruck O., et. al, 2014, J. Geod., 89(3):283-297.

Mouret S., 2011, Phys. Rev. D 84, 122001.

Müller H., et al, 2008, Phys. Rev. Letters 100, 031101.

Neal R.M., 2003, Ann. Statist., 31(3):705-767.

Nunes N., J. Lidsey, 2004, Phys. Rev. D 69, 123511.

Origlia S., et al., 2016, arXiv: 1603.06062.

Overduin J., P. Wesson, 1997, Phys. Report 283, 303.

Peil S., et al, 2013, Phys. Rev. A 87, 010102(R).

Perez P., Y. Sacquin, 2012, Class. And Quantum Grav. 29, 184008.

Peters A. et al, 1999, Nature 400, 849.

Peters A., et al, 2001, Metrologia 38, 25.

Pitjeva E., and N. Pitjev, 2013, MNRAS 432, 3431.

Planck Collaboration, 2013, A \& A 571, A16.

Pound R., G. Rebka, 1959, Phys. Rev. Letters 3, 439.

Prange L., et al., 2013, in P Willis, editor, IAG Potsdam 2013 Proceedings. Springer, International Association of Geodesy Symposia, 2014.

Ratra B., P. Peebles, Phys. Rev. D 37, 3406.

Reinhardt S., et al, 2007, Nature Physics 3, 861.

Robertson H., 1949, Rev. of Modern Phys. 21, 378.

Rochat P., et al., 2012, in Proceedings of 2012 European Navigation Conference, 25-27 Apr. 2012, Gdansk (Poland).

Rosenband T., et al, 2008, Science 319, 1808.

Saathoff G., et al, 2003, Phys. Rev. Letters 91, 190403.

Schlamminger S., et al, 2008, Phys. Rev. Letters 100, 041101.

Shao L., 2014, Phys. Rev. Letters 112, 111103.

Srianand R., et al, 2004, Phys. Rev. Letters 92, 121302.

Stadnik Y., and V. Flambaum, 2014b, Phys. Rev. Letters 114, 161301.

Stadnik Y., V. Flambaum, 2015, Phys. Rev. Letters 115, 201301.

Stanwix P., et al, 2006, Phys. Rev. D 74, 081101 (R).

STE-QUEST, 2013, Assessment study report, ESA report ESA/SRE 6, (2013). Available on http://sci.esa.int/ste-quest/53445-ste-quest-yellow-book/.

Stubbs C., 1993, Phys. Rev. Letters 70, 119.

Swaters R., et al, 2010, ApJ 718, 380.

Talmadge C., et al, 1988, Phys. Rev. Letters 61, 1159.

Tarallo M., et al, 2014, Phys. Rev. Letters 113, 023005.

Tasson J., 2014, Rep. on Progress in Phys. 77, 6.

Thorne K., Lee D., Lightman A., 1971, Phys. Rev. D 7, 3563.

Turyshev S., 2009, Physics Uspekhi 52, 1.

Uzan J.-P., 2011, LRR 14,2.

Van Tilburg K., et al, 2015, Phys. Rev. Letters 115, 011802.

Verma A., et al, 2014, A\&A 561, A115.

Vessot R.F.C., and M. W. Levine, 1979, General Relativity and Gravitation, 10:181-204.

Vessot R.F.C., et al, 1980, Phys. Rev. Letters 45, 2081.

Vessot R.F.C., et al, 1989, Advances in Space Research, 9:21-28. 
Wang J., L. Hui, J. Khoury, 2012, Phys. Rev. Letters 109, 241301.

Webb J., et al, 2003, Phys. Rev. Letters 87, 091301.

Webb J., et al, 2011, Phys. Rev. Letters 107, 191101.

Will C., 1993, Theory and Experiment in Gravitational Physics, Cambridge University Press.

Will C., 2014, Living Review in Relativity 17, 4.

Williams J., et al, 2009, IJMPD 18, 1129

Williams J., et al, 2012, Class. And Quantum Grav. 29, 184004.

Wolf P. et al, 2003, Phys. Rev. Letters 90, 060402.

Wolf P., et al, 2006, Phys. Rev. Letters 96, 060801.

Wolf P., G. Petit, 1997, Phys. Rev. A 56, 4405.

Wolf P., et al., 2006, Phys. Rev. Lett., 96:060801.

Zhou L. et al, 2015, Phys. Rev. Letters 115, 1.

Zlatev I., et al, 1999, Phys. Rev. Letters 82, 896.

Zucker S., et al, 2006, ApJ 639, L21. 